\title{
Asteroid Systems: Binaries, Triples, and Pairs
}

\author{
Jean-Luc Margot \\ University of California, Los Angeles \\ Petr Pravec \\ Astronomical Institute of the Czech Republic Academy of Sciences \\ Patrick Taylor \\ Arecibo Observatory \\ Benoît Carry \\ Institut de Mécanique Céleste et de Calcul des Éphémérides \\ Seth Jacobson \\ Côte d'Azur Observatory
}

\begin{abstract}
In the past decade, the number of known binary near-Earth asteroids has more than quadrupled and the number of known large main belt asteroids with satellites has doubled. Half a dozen triple asteroids have been discovered, and the previously unrecognized populations of asteroid pairs and small main belt binaries have been identified. The current observational evidence confirms that small $(\lesssim 20 \mathrm{~km})$ binaries form by rotational fission and establishes that the YORP effect powers the spin-up process. A unifying paradigm based on rotational fission and post-fission dynamics can explain the formation of small binaries, triples, and pairs. Large ( $\gtrsim 20$ $\mathrm{km}$ ) binaries with small satellites are most likely created during large collisions.
\end{abstract}

\section{INTRODUCTION}

\subsection{Motivation}

Multiple-asteroid systems are important because they represent a sizable fraction of the asteroid population and because they enable investigations of a number of properties and processes that are often difficult to probe by other means. The binaries, triples, and pairs inform us about a great variety of asteroid attributes, including physical properties, composition, interior structure, formation processes, and evolutionary processes.

Observations of binaries and triples provide the most powerful way of deriving reliable masses and densities for a large number of objects. The density measurements help us understand the composition and internal structure of minor planets. Binary systems offer opportunities to measure thermal and mechanical properties, which are generally poorly known.

The binary and triple systems within nearEarth asteroids (NEAs), main belt asteroids (MBAs), and trans-Neptunian objects (TNOs) exhibit a variety of formation mechanisms (Merline et al. 2002c; Noll et al. 2008). As such, they provide an invaluable window on accretional, collisional, tidal, and radiative processes that are critical in planet formation. The distribution and configurations of the multiple-asteroid systems also provide a rich array of constraints on their environment, their formation, and their evolutionary pathways.

Observations rely primarily on ground-based 
telescopes and the Hubble Space Telescope (HST). For an up-to-date list of binaries and triples in the solar system, see Johnston (2014). We describe observational techniques only briefly because this material is available elsewhere (e.g., Merline et al. 2002c). A few emerging techniques will be described in more detail. Likewise, we refer the reader to other texts for an extensive history of the field (e.g., Merline et al. 2002c) and highlight only a few of the developments here.

\subsection{History}

Early search programs for asteroid satellites were unsuccessful, returning negative or dubious results, such that the authors of the Asteroids II review chapter chose the prudent title "Do asteroids have satellites?" (Weidenschilling et al. 1989). The chapter provides an excellent discussion of the physics of several formation mechanisms that were postulated at the time. The perspective changed with the flyby of (243) Ida by the Galileo spacecraft in 1993 and the discovery of its small satellite Dactyl (Chapman et al. 1995; Belton et al. 1995). Ground-based efforts intensified and resulted in the discovery of a satellite around (45) Eugenia by Merline et al. (1999). Several other discoveries followed in rapid succession. The relatively small sizes of the MBA satellites suggested formation in sub-catastrophic or catastrophic collisions (Durda 1996; Doressoundiram et al. 1997).

The discovery of MBA satellites, coupled with analysis of terrestrial doublet craters (Bottke and Melosh 1996a,b) and anomalous lightcurve observations (Pravec and Hahn 1997), suggested the existence of binary asteroids in the near-Earth population as well. The unambiguous detection of five NEA binaries by radar cemented this finding and indicated that NEA satellites form by a spin-up and rotational fission process (Margot et al. 2002). Lightcurve observers reached the same conclusion independently (Pravec and Harris 2007). Both radar and lightcurve observations revealed that, far from being rare, binary asteroids are relatively common (Pravec et al. 1999; Margot et al. 2002; Pravec et al. 2006). By the time the Asteroids III review chapter was written, a more decisive title ("Asteroids do have satellites") had become appropriate (Merline et al. 2002c). This review focuses on the developments that followed the publication of Asteroids III.

\subsection{Terminology}

Two- and three-component asteroids that are gravitationally bound will be referred to as binary asteroids (or binaries) and triple asteroids (or triples), respectively. (Triple is favored over the more directly analogous terms trinary and ternary because of long-established usage in astronomy). Asteroid pairs denote asteroid components that are genetically related but not gravitationally bound. Paired binaries or paired triples are asteroid pairs where the larger asteroid is itself a binary or triple asteroid. The larger component in binaries, triples, and pairs is referred to as the primary component or primary. The smaller component in binaries is referred to as the secondary component or secondary.

There has been some confusion in the literature about the meaning of the word "asynchronous." Here, we adopt the terminology proposed by Margot (2010) and later implemented by Jacobson and Scheeres (2011b) and Fang and Margot (2012c). Binaries with an absence of spin-orbit synchronism are called asynchronous binaries. Binaries with a secondary spin period synchronized to the mutual orbit period are called synchronous binaries. Binaries with both primary and secondary spin periods synchronized to the mutual orbit period are called doubly synchronous binaries. If generalization to systems with more than one satellite is needed, we affix the terms synchronous and asynchronous to the satellites being considered.

It is useful to present results for small and large asteroids. We place an approximate dividing line at the size at which objects are substantially affected by the YORP effect during their lifetime. For typical NEAs and MBAs, this dividing line corresponds to a diameter of about $20 \mathrm{~km}$ (Jacobson et al. 2014a). We define very small asteroids as those with diameters of less than $200 \mathrm{~m}$. This is the approximate size below which many asteroids are observed to spin faster than the disruption 
rate of a body with no shear or tensile strength $\omega_{d}=\sqrt{4 \pi \rho G / 3}$, where $\rho$ is the density and $G$ is the gravitational constant.

We use two additional acronyms. The YORP effect is a radiation-powered rotational acceleration mechanism for small asteroids (Rubincam 2000). The binary YORP (BYORP) effect is a radiation-powered acceleration mechanism that may expand or contract the orbits of some synchronous asteroids (Ćuk and Burns 2005).

\section{OBSERVATIONS}

Several observational techniques are available for discovering, detecting, and studying binaries, triples, and pairs, each with its strengths and weaknesses. This section describes recent results and illustrates the complementarity of the observational techniques that characterize individual asteroid systems and entire populations.

\subsection{Radar Observations of NEA Systems}

Radar has proven to be a powerful method of detecting secondaries to NEAs, enabling the discovery (as of September 2014) of the satellites in $71 \%$ of the 49 known multiple-component NEA systems, including 33 of 47 binaries and both undisputed triple systems. Of the 14 binary NEAs discovered via optical lightcurve techniques, 6 have been confirmed with follow-up radar observations during later apparitions. Overall, radar detections suggest that about one in six NEAs larger than $200 \mathrm{~m}$ in diameter are multipleasteroid systems (Margot et al. 2002; Taylor et al. 2012a), though $200 \mathrm{~m}$ is not a sharp cutoff. Three binary NEA systems identified by radar have primary components with suggested diameters of $120 \mathrm{~m}$ to $180 \mathrm{~m}: 2003 \mathrm{SS}_{84}$ (Nolan et al. 2003), (363599) $2004 \mathrm{FG}_{11}$ (Taylor et al. 2012c), and $1994 \mathrm{CJ}_{1}$ (Taylor et al. 2014). For comparison, the largest primaries of binary NEAs imaged with radar: (5143) Heracles (Taylor et al. 2012b), the possible triple (276049) $2002 \mathrm{CE}_{26}$ (Shepard et al. 2006), and (285263) $1998 \mathrm{QE}_{2}$ (Springmann et al. 2014), are more than an order of magnitude larger at $>3 \mathrm{~km}$ in diameter. It is likely that $\sim 8 \mathrm{~km}$ diameter (1866) Sisyphus has a secondary based on analysis of frequency-only observations obtained on four separate dates in 1985 (Ostro, pers. comm., 2001).

Radar observations can be used to detect asteroid satellites because of the ability to resolve the components of the system both spatially (along the observer's line of sight) and in terms of frequency (due to Doppler shifts from the rotational and orbital line-of-sight velocities), resulting in a measurable separation between the components in two dimensions. Direct detection of a satellite in frequency-only spectra or radar images typically occurs within one observing session and often within minutes of observation. The bandwidth of the echo of a component scales directly with the diameter and rotation rate. Thus, in a frequency-only experiment, the signal of the smaller, relatively slowly rotating satellite is condensed to a smaller bandwidth that is superimposed upon the broadband signal of the larger, often rapidly rotating, primary (Fig. 1, top). Not all radar-observed binaries present this characteristic spectrum (e.g., where the secondary spins faster than the synchronous rate), but all are readily detected in radar images when the components are also resolved spatially (Fig. 1, bottom). Because the spatial resolution achieved with radar instruments corresponds to an effective angular resolution of better than $\sim 1$ milliarcsecond (mas), there is no bias against the detection of satellites orbiting very close to the primary component. Multiple measurements of the range and frequency separations of the components over days of sky motion provide the geometric leverage required to determine the orbit of the secondary around the primary. This can be done for any orbital orientation and yields the total system mass, a property that is difficult to estimate otherwise. Other techniques involve analyzing spacecraft flyby and orbit trajectories (e.g., Yeomans et al. 1999), measuring the Yarkovsky orbital drift in conjunction with thermal properties (e.g., Chesley et al. 2014), or observing the gravitational perturbations resulting from asteroid encounters (e.g., Hilton 2002).

Most binary NEA systems observed to date have a rapidly rotating primary and a smaller 

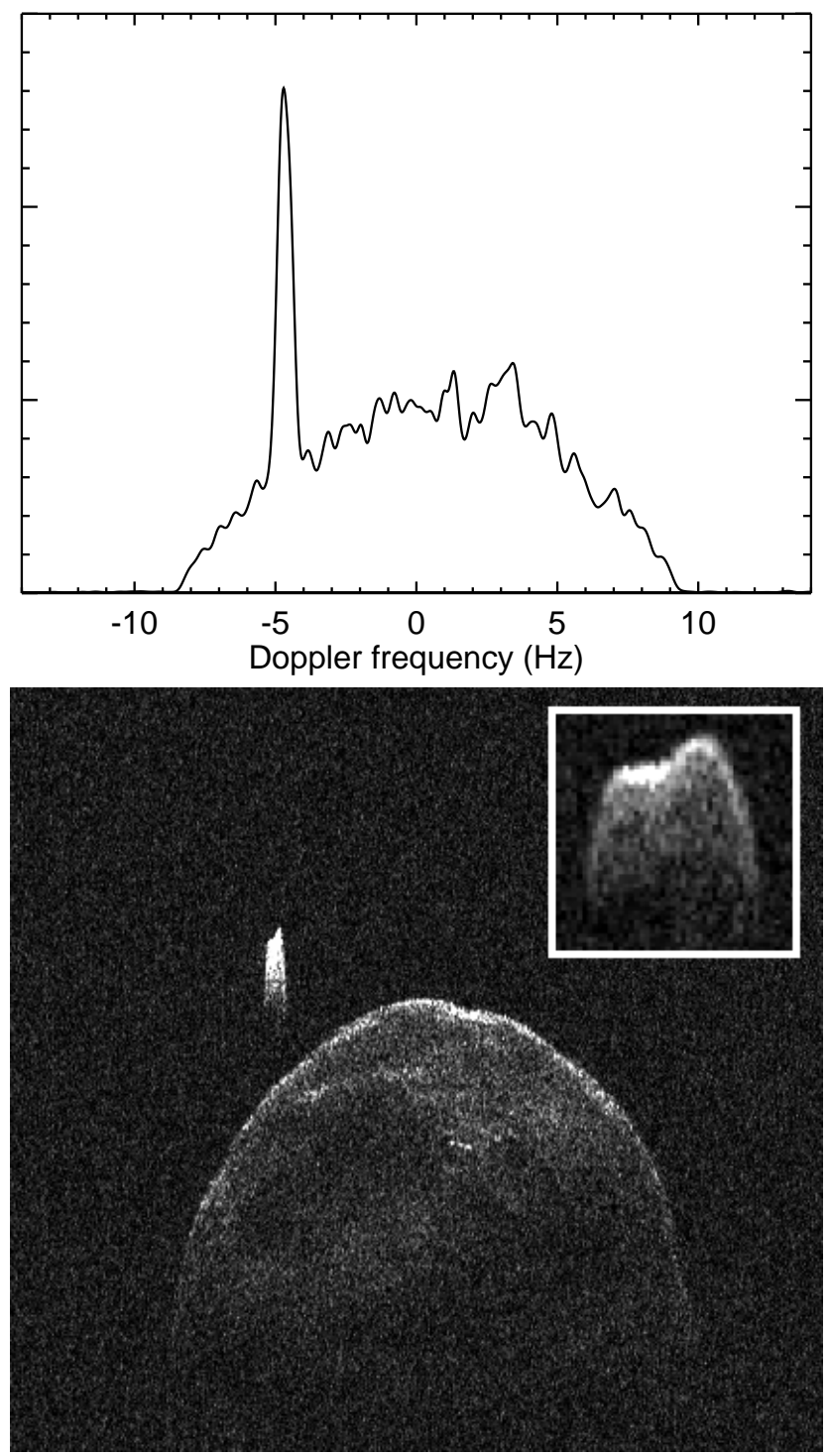

Fig. 1. - Binary near-Earth asteroid (285263) 1998 $\mathrm{QE}_{2}$ as detected using the Arecibo planetary radar system. In the frequency-only spectrum showing echo power as a function of Doppler frequency (top), the narrowband echo of the tidally locked secondary stands out against the broadband echo of the larger, faster-rotating primary. In the radar image (bottom), the components are spatially resolved $(7.5 \mathrm{~m} /$ pixel). The vertical axis represents distance from the observer increasing downward. The horizontal axis is Doppler frequency due to the orbital and rotational motion of the components. Note that if one summed the pixel values in each column of the image, the intensity as a function of Doppler frequency would approximate the spectrum above. The secondary is roughly onefourth the size of the primary (measured in the vertical dimension), though the Doppler breadth of the primary gives the illusion of a greater size disparity. The shape of the secondary (inset) is distinctly nonspherical when viewed with finer frequency resolution. secondary of order a few tenths the size of the primary (a secondary-to-primary mass ratio of roughly 0.001 to 0.1 ), whose rotation is synchronized to the mutual orbit period. The majority of primaries rotate in less than $2.8 \mathrm{~h}$, though they range from $2.2593 \mathrm{~h}$ for (65803) Didymos (Pravec et al. 2006) to $4.749 \mathrm{~h}$ for 1998 $\mathrm{QE}_{2}$ (P. Pravec, pers. comm., 2013). The known outlier is the nearly equal-mass binary (69230) Hermes, whose components both appear to have $13.894 \mathrm{~h}$ periods synchronized to their mutual orbit period (Margot et al. 2006). This doubly synchronous configuration is most likely due to rapid tidal evolution (Taylor and Margot 2011). While the rotations of satellites in NEA binaries tend to be tidally locked to their orbital mean motions with periods typically within a factor of two of 24 $\mathrm{h}$ (often resulting in the characteristic appearance shown in Fig. 1), about one in four radar-observed multiple-asteroid systems have an asynchronous satellite (Brozović et al. 2011), all of which rotate faster than their orbital rate. Well-studied examples include (35107) 1991 VH (Naidu et al. 2012), (153958) $2002 \mathrm{AM}_{31}$ (Taylor et al. 2013), (311066) 2004 DC (Taylor et al. 2008), and the outer satellites of both undisputed triple systems (153591) $2001 \mathrm{SN}_{263}$ (Nolan et al. 2008; Fang et al. 2011; Becker et al. 2015) and (136617) 1994 CC (Brozović et al. 2011; Fang et al. 2011). Of the known asynchronous satellites, all have wide component separations ( $>7$ primary radii), translating to longer-than-typical orbital periods, and/or eccentric orbits $(>0.05)$, that are either remnants of their formation mechanism or products of subsequent dynamical evolution (Fang and Margot 2012c).

The shortest orbital periods detected with radar so far are those of Didymos and $2006 \mathrm{GY}_{2}$ with $P_{\text {orb }}=11.90_{-0.02}^{+0.03} \mathrm{~h}$ and $11.7 \pm 0.2 \mathrm{~h}$, respectively (Benner et al. 2010; Brooks 2006). For Didymos, the semi-major axis is $a=1.18_{-0.02}^{+0.04} \mathrm{~km}$, just outside the classical fluid Roche limit of $\sim 1$ $\mathrm{km}$ for equal-density components. Other systems with satellites orbiting near this limit include $2002 \mathrm{CE}_{26}$ and $2001 \mathrm{SN}_{263}$. The significance of this limit is unclear, as $\sim 100 \mathrm{~m}$ secondaries with a cohesion comparable to comet regolith or sand can likely survive on orbits interior to the Roche 
limit (Taylor and Margot 2010, and references therein).

Inversion of a series of radar images can provide a three-dimensional shape model and complete spin-state description given sufficient signal, resolution, and orientational coverage (Hudson 1993; Magri et al. 2007). Shape reconstruction of the larger component of (66391) $1999 \mathrm{KW}_{4}$ (Ostro et al. 2006) demonstrated that the canonical shape of an NEA primary has a characteristic circular equatorial bulge, uniformly sloped sides, and polar flattening akin to a spinning top. Such a shape is shared by the primaries of 2004 DC, $1994 \mathrm{CC}, 2001 \mathrm{SN}_{263}$, and (185851) $2000 \mathrm{DP}_{107}$ (Naidu et al. 2015), though some primaries have less pronounced equatorial belts, e.g., $2002 \mathrm{CE}_{26}$ and $1998 \mathrm{QE}_{2}$. Some single asteroids have a similar shape, e.g., (101955) Bennu (Nolan et al. 2013) and (341843) $2008 \mathrm{EV}_{5}$ (Busch et al. 2011), but do not have satellites, possibly because one has not yet formed or has been lost in the past. Shape model renditions are shown in Benner et al. (this volume). Often the resolution of radar images of the smaller satellites is insufficient for shape inversion, but radar images suggest that the satellites are typically elongated, e.g., $2000 \mathrm{DP}_{107}$, $1999 \mathrm{KW}_{4}, 2001 \mathrm{SN}_{263}, 1991 \mathrm{VH}$, and $1998 \mathrm{QE}_{2}$.

Shapes and volumes obtained from inversion of radar images, combined with the system mass derived from the orbital motion observed in radar images, provide the density of the system (or of the individual components if the mass ratio is measurable from reflex motion). Low densities of order $1 \mathrm{~g} / \mathrm{cm}^{3}$ (Shepard et al. 2006; Becker et al. 2015) to $2 \mathrm{~g} / \mathrm{cm}^{3}$ (Ostro et al. 2006; Brozović et al. 2011) suggest significant internal macroporosity of order 50\%, implying a rubblepile internal structure for the components. At such low densities, the rapid rotation of the primary places particles along the equatorial belt in a near-weightless environment. The combination of rapid rotation, shape, and implied porosity and rubble-pile structure has implications for the formation mechanism of small multiple-asteroid systems (Section 4).

While radar allows for direct, unambiguous de- tection of asteroid satellites, its range is limited. Because radar requires the transmission and reception of a signal, the strength of the received signal falls as the fourth power of the distance to the target and, thus, is best suited for detecting multiple-component systems passing within $\sim 0.2$ astronomical units (au) of Earth. Satellites in the main asteroid belt simply tend to be too small and too far away to detect with present radar capabilities and require application of different observational techniques.

\subsection{Lightcurve Observations of NEA and Small MBA Systems}

A photometric lightcurve is a time series of measurements of the total brightness of an asteroid. Detections of binary asteroids by photometric lightcurve observations utilize the fact that the components can obscure or cast a shadow on one another, producing occultations or eclipses, respectively. The attenuations can be used to both reveal and characterize binaries (Fig. 2). The observational, analysis, and modeling techniques were described in Pravec et al. (2006); Scheirich and Pravec (2009); Scheirich et al. (2015).

Early reports (Tedesco 1979; Cellino et al. 1985) of asteroids suspected to be binaries on the basis of anomalous lightcurves (including (15) Eunomia, (39) Laetitia, (43) Ariadne, (44) Nysa, (49) Pales, (61) Danae, (63) Ausonia, (82) Alkmene, (171) Ophelia, and (192) Nausikaa) have remained largely unconfirmed despite extensive follow-up searches. The first serious candidate for detection with this technique was NEA (385186) $1994 \mathrm{AW}_{1}$ (Pravec and Hahn 1997), whose binary nature was confirmed by photometric observations in 2008 (Birlan et al. 2010). Since 1997, nearly 100 binaries among near-Earth and small main belt asteroids have been detected with the photometric method. The binary asteroid database constructed by Pravec and Harris (2007) (http://www.asu.cas.cz/ asteroid/binastdata.htm) includes data for $86 \mathrm{MBA}$ and NEA binaries that were securely detected by photometry and for which basic parameters have been derived, such as the primary spin period, the orbital period, and the primary-to-secondary mean diameter ratio. A 


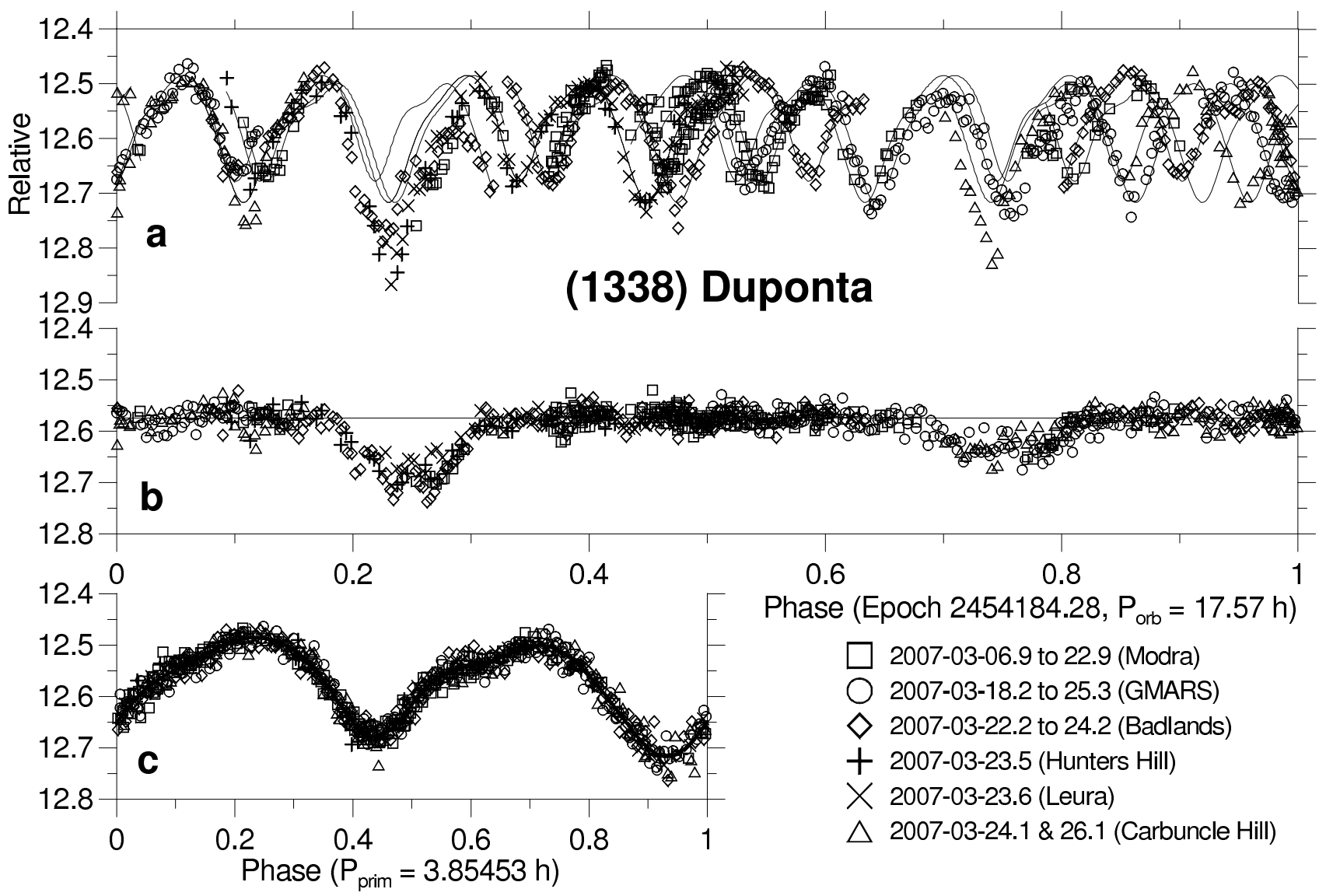

Fig. 2.- Lightcurve data of (1338) Duponta, which has a secondary-to-primary diameter ratio of about 0.24. (a) The original data showing both lightcurve components, folded with the orbit period. (b) The orbital lightcurve component, derived after subtraction of the primary lightcurve component, showing the mutual events between components of the binary system. (c) The primary lightcurve component. Figure from Pravec et al. (2012).

few tens of additional MBAs and NEAs are suspected to be binaries and await confirmation with more detailed observations in the future.

Among the main findings obtained from photometric observations is that binary asteroids are ubiquitous. They have been found among NEAs, Mars-crossers (MCs), and throughout the main belt, both among asteroids that have been identified as family members and among asteroids that have not. Pravec et al. (2006) derived the fraction of binaries among NEAs larger than 300 meters to be $15 \pm 4 \%$. A binary fraction among MBAs has not been derived precisely due to less wellcharacterized observational selection effects, but their photometric discovery rate is similar to the discovery rate of binaries among NEAs. Thus, binaries are suspected to be as frequent among MBAs as they are among NEAs. There appears to be an upper limit on the primary diameter for photometrically detected binaries of about $13 \mathrm{~km}$; the largest detected binary is (939) Isberga with $D_{p}=13.4 \pm 1.3 \mathrm{~km}$ (Carry et al. 2015). A lower size limit on the primary diameter $D_{p}$ is less clear. The smallest detected binary is $2000 \mathrm{UG}_{11}$ with $D_{p}=0.26 \pm 0.03 \mathrm{~km}$ (Pravec et al. 2006), but smaller binaries are known to exist (Section 2.1). Their absence in lightcurve data sets may be due in part to a bias against detecting small binaries in the initial surveys.

Another key finding is that small binary asteroids have, with only two or three exceptions, a near-critical angular momentum content (Fig. 3). As shown by Pravec and Harris (2007), their angular momentum is consistent with formation by fission of critically spinning parent bodies of a cohesionless, rubble pile structure. The exceptions 


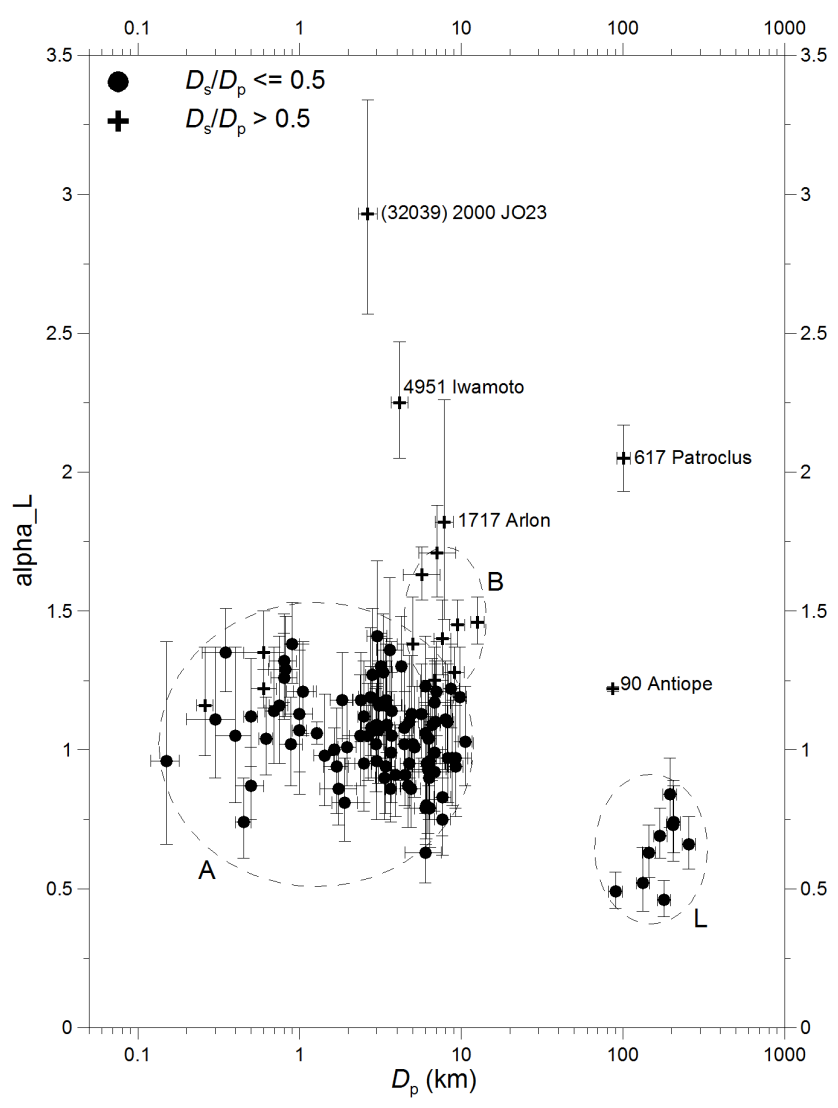

Fig. 3.- Estimated values of the normalized total angular momentum content of binaries versus primary diameter. The quantity $\alpha_{L}$ is the sum of orbital and spin angular momenta normalized by the angular momentum of an equivalent sphere spinning at the critical disruption spin rate $\omega_{d}=\sqrt{4 \pi \rho G / 3}$ where $\rho$ is the density and $G$ is the gravitational constant. In the Darwin notation, $\alpha_{L}=1$ corresponds to $J / J^{\prime}=0.4$. Group A contains small NEA, MC, and MBA binaries. Group B consists of doubly synchronous small MBAs with nearly equal-size components. Group L represents large MBAs with small satellites (Section 2.5). Two exceptional cases are the doubly synchronous asteroids (90) Antiope and (617) Patroclus (Section 2.5). Figure updated from Pravec and Harris (2007).

are the semi-wide systems (32039) $2000 \mathrm{JO}_{23}$ and (4951) Iwamoto, and possibly also (1717) Arlon, with orbital periods of $117 \mathrm{~h}$ to $360 \mathrm{~h}$ and supercritical total angular momentum content.

The orbital poles of main belt binaries were found to have a highly anisotropic distribution, concentrating within 30 degrees of the poles of the ecliptic (Pravec et al. 2012). The preferential orientations of the orbital poles suggest that their parent bodies or the primaries were tilted by the YORP effect towards the asymptotic spin states near obliquities 0 and 180 degrees, consistent with observations of single asteroids (Hanuš et al. 2011).

Another significant finding is that there appears to be a lower limit on the separation between components of binary systems of about $a / D_{p}=1.5$, corresponding to an orbital period of 11-12 h for typical densities. Lightcurve observations indicate that the orbital period of Didymos is $P_{\text {orb }}=11.91 \pm 0.02 \mathrm{~h}$ (Pravec et al. 2006), consistent with the radar estimate. This suggests an orbit close to the Roche limit for strengthless satellites (but see prior remark about orbits interior to the Roche limit).

Photometric observations of a binary system over multiple apparitions can be used to detect a change in the separation of the components due to the effect on mutual event timing. An extensive set of photometric observations of the synchronous binary (175706) $1996 \mathrm{FG}_{3}$ obtained during 1996-2013 places an upper limit on the drift of its semi-major axis that is one order of magnitude less than estimated on the basis of the BYORP theory (Scheirich et al. 2015). This system may be in an equilibrium between BYORP and tidal torques as proposed for synchronous binary asteroids by Jacobson and Scheeres (2011a).

Some data sets strongly suggest the presence of triple asteroids. In these cases, an additional rotational component that does not belong to the primary or the close eclipsing secondary is present in the lightcurve. This additional rotational component does not disappear during mutual events where the eclipsing close secondary is obscured by or in the shadow of the primary. Pravec et al. (2012) identified three such cases: (1830) Pogson, (2006) Polonskaya, and (2577) Litva. The latter has been confirmed by direct imaging observations of the third body (second satellite) on a wide orbit (Merline et al. 2013).

Other data sets reveal the existence of paired binaries/triples. Two such cases have been published: the pair composed of (3749) Balam and 2009 BR $_{60}$ (Vokrouhlický 2009, and references therein) and the pair composed of (8306) Shoko 
and 2011 SR $_{158}$ (Pravec et al. 2013). Balam is a confirmed triple, with a distant satellite detected by direct imaging (Merline et al. 2002a) and a close satellite detected by lightcurve observations (Marchis et al. 2008d). Shoko is a suspected triple as well: Using lightcurve observations, Pravec et al. (2013) detected an eclipsing, synchronous close satellite with $P_{\text {orb }}=36.2 \mathrm{~h}$ and a third rotational component attributed to an outer satellite.

While the population of binary NEAs and small MBAs is composed primarily of synchronous systems, and secondarily of asynchronous systems with low secondary-to-primary size ratios $\left(D_{s} / D_{p}<0.5\right)$, doubly synchronous binaries with nearly equal-size components also exist (Fig. 4). Nine such systems with $D_{s} / D_{p}>0.7$ and orbital periods between $15 \mathrm{~h}$ and $118 \mathrm{~h}$ have been reliably identified in the main belt (e.g., Behrend et al. 2006; Kryszczyńska et al. 2009, see also the Pravec and Harris binary database described above).

Another important observation is that, with the exception of doubly synchronous systems, all binaries have unelongated, near-spheroidal primary shapes, as evidenced by their low primary amplitudes not exceeding $0.3 \mathrm{mag}$ (when corrected to zero phase angle). This suggests that their primaries may have shapes similar to the toplike shapes that have been observed for 1999 $\mathrm{KW}_{4}$ (Ostro et al. 2006) and several other binaries by radar.

All the properties revealed by photometric observations indicate that binary systems among NEAs and small MBAs were formed from critically spinning cohesionless parent bodies, with YORP as the predominant spin-up mechanism. This finding is consistent with the fact that the observed $0.2-13 \mathrm{~km}$ size range of binaries corresponds to the size range where the spin barrier against asteroid rotations faster than about $2.2 \mathrm{~h}$ has been observed (e.g., Pravec et al. 2007).

Although lightcurve observations provide powerful constraints on binaries, there are limitations. Detection of mutual events requires an edge-on geometry and observations at the time of the events, such that some binaries remain unde-

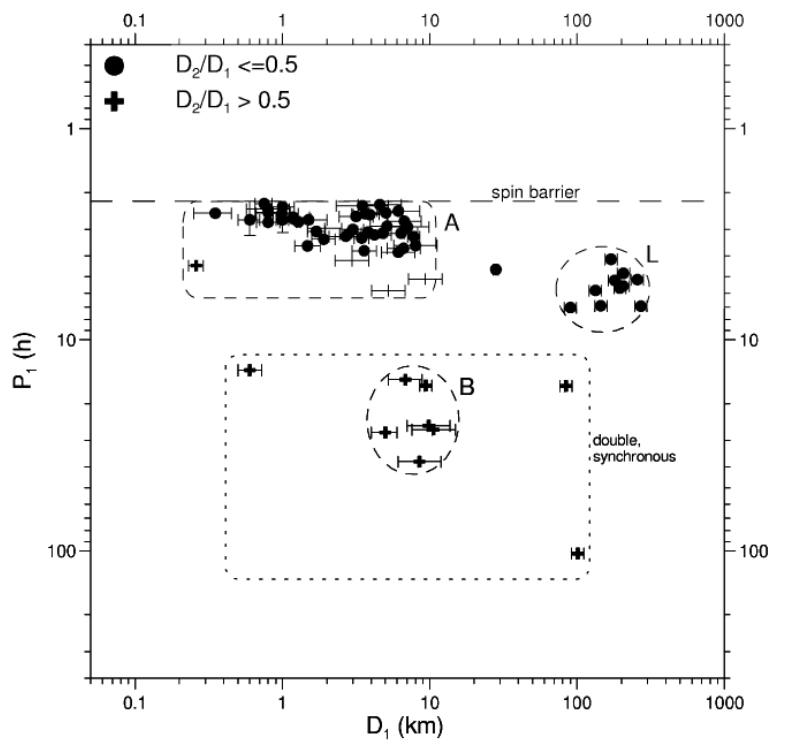

Fig. 4.- Primary rotation period versus primary diameter. Groups A, B, and L are defined in the caption of Fig. 3. Three doubly synchronous asteroids with nearly equal-size components lie isolated in the plot: (69230) Hermes on the left and (90) Antiope and (617) Patroclus on the right of group B. Note that members of group A cluster near the disruption spin limit for strengthless bodies. Figure from Pravec and Harris (2007).

tected (e.g., (69230) Hermes during its 2003 apparition). Small satellites also escape detection because their effect on the lightcurve is not measurable (e.g., satellites with $D_{s} / D_{p} \lesssim 0.17$ remain undetected if the minimum detectable relative brightness attenuation is $\sim 0.03 \mathrm{mag}$ ). The probability of mutual event detection is larger at smaller semi-major axes (expressed in units of primary radius) and at larger size ratios, resulting in observational biases (e.g., Pravec et al. 2012). Finally, lightcurve observations yield relative, not absolute, measurements of orbital separations. Detection of small or distant secondaries and direct measurement of orbital separation must instead rely on other observational techniques.

\subsection{Lightcurve Observations of Asteroid Pairs}

Vokrouhlický and Nesvorný (2008) reported evidence for pairs of MBAs with bodies in each pair having nearly identical heliocentric orbits. Because chance associations can be ruled out, 
the asteroids in each pair must be genetically related. Quantifying the difference in orbital parameters is accomplished with a metric $d$ that corresponds roughly to the relative velocity between the bodies at close encounter. Vokrouhlický and Nesvorný (2008) identified 44 asteroid pairs (excluding family members) with a distance between the orbits of their components amounting to $d<10 \mathrm{~m} / \mathrm{s}$. They showed that, when integrated backwards in time, the orbits converge at a certain moment in the past with a physical distance much less than the radius of the Hill sphere and with a low relative velocity on the order of $1 \mathrm{~m} / \mathrm{s}$.

Pravec and Vokrouhlický (2009) developed a method to identify probable asteroid pairs by selecting candidate pairs with a similar distance criterion, then computing the probability that each candidate pair emerged as a result of a coincidence between two unrelated asteroids. They identified 72 probable asteroid pairs, reproducing most of the 44 previously known pairs. Most of the new candidates were later confirmed to be real pairs using backward integrations of their heliocentric orbits.

Vokrouhlický and Nesvorný (2008) proposed a few possible formation mechanisms for the asteroid pairs: collisional disruption, rotational fission, and splitting of unstable asteroid binaries. Pravec et al. (2010) conducted a survey of the rotational properties of asteroid pairs, and they found a strong correlation between the primary rotational periods and the secondary-to-primary mass ratio (Fig. 5). They showed that this correlation fits precisely with the predictions of a model by Scheeres (2007) in which a parent body with zero tensile strength undergoes rotational fission. The model predicts that primaries of low mass ratio pairs ( $q \lesssim 0.05$ ) have not had their spin substantially slowed down in the separation process and should rotate rapidly with frequencies close to the fission spin rate. The observed periods are between 2.4 and $5 \mathrm{~h}$. Primaries of medium mass ratio pairs $(q=0.05$ to $\sim 0.2)$ have had their spin slowed down according to the model because a substantial amount of angular momentum was taken away by the escaped secondary. This trend

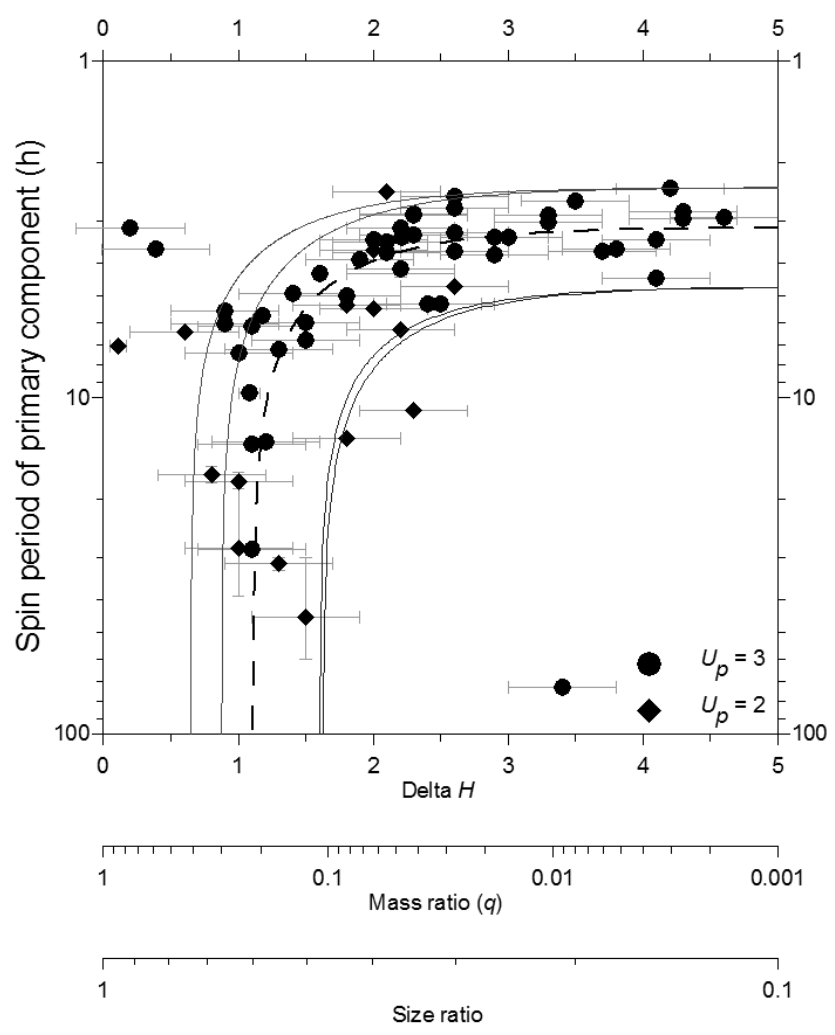

Fig. 5.- Primary rotation periods versus mass ratios of asteroid pairs. The mass ratio values were estimated from the differences between the absolute magnitudes of the pair components, $\Delta H$. Circles are data points with quality code rating $U_{p}=3$, meaning a precise period determination. Diamonds are data points with $U_{p}=2$, which are somewhat less certain estimates. Error bars are one standard deviation. The data match the predictions (curves) of a model of rotational fission with a few adjustable parameters. In the model, $A_{\text {ini }}$ is the binary system's initial orbit semi-major axis, $\alpha_{L}$ is the normalized total angular momentum of the system (Fig. 3), and $a_{p}, b_{p}, c_{p}$ are the long, intermediate, and short axis of the dynamically equivalent equal mass ellipsoid of the primary. All models shown assume $b_{p} / c_{p}=1.2$. The dashed curve shows the best-fit model with $\alpha_{L}=1.0, a_{p} / b_{p}=1.4$ and $A_{\text {ini }} / b_{p}=3$. Solid curves represent upper and lower limiting cases with $\alpha_{L}=0.7-1.2$. Figure updated from Pravec et al. (2010).

is observed in the data (Fig. 5). Finally, high mass ratio pairs with $q>0.2$ should not exist, as the free energy in the proto-binary system formed by rotational fission would be negative and the components would be unable to separate. Observations mostly corroborate this prediction: all 32 
pairs in the sample of Pravec et al. (2010) were found to have a mass ratio $\lesssim 0.2$. However, an expanded photometric survey with 64 asteroid pairs observed between 2012 and the date of this writing reveals 3 pairs with high mass ratio $(q>0.5)$. Their formation requires an additional supply of angular momentum. Another important finding by Pravec et al. (2010) is that the primaries of asteroid pairs have lightcurve amplitudes that imply shapes with a broad range of elongations, i.e., unlike the primaries of binaries (Sections 2.1 and 2.2), the primaries of asteroid pairs do not tend to be nearly spheroidal.

\subsection{Spectral Observations of Asteroid Pairs}

Colorimetric and spectral observations of about 20 asteroid pairs indicate that members of an asteroid pair generally have similar spectra (Duddy et al. 2012; Moskovitz 2012; Duddy et al. 2013; Polishook et al. 2014a; Wolters et al. 2014). In some pairs, the authors observed subtle spectral differences between the components and attributed them to a larger amount of weathered material on the surface of the primary. In two pairs, they observed somewhat more significant spectral differences. For the pair (17198)-(229056), both Duddy et al. (2013) and Wolters et al. (2014) found that the primary is redder, i.e., it has a somewhat higher spectral slope than the secondary in the observed spectral range $0.5-0.9 \mu \mathrm{m}$. It is unclear why their spectra differ despite a strong dynamical link between the two asteroids. For the pair (19289)-(278067), Wolters et al. (2014) observed a spectral difference similar to that seen in (17198)-(229056), but Duddy et al. (2013) observed very similar spectra. Crossvalidation of the methods or additional observations, perhaps rotationally resolved, are needed to resolve the discrepancy.

\subsection{Direct Imaging of MBA and Trojan Sys- tems}

Direct imaging of asteroids can reveal the presence of satellites and, following the long tradition of orbit determination of binary stars and planetary satellites, lead to estimates of orbital parameters (Fig. 6). This observing mode remains challenging because the satellites are generally much smaller and fainter than their respective primaries and because most satellites known to date orbit at angular separations below 1 arcsecond. Satellite discoveries have therefore followed the development of adaptive optics ( $\mathrm{AO}$ ), and recent advances have enabled the detection of asteroid satellites that had remained undetected in prior searches.

Instruments must have sufficient contrast and resolving power to detect asteroid satellites with direct imaging. For a 50-100 km diameter asteroid in the main belt orbited by a satellite a few $\mathrm{km}$ across, the typical angular separation is generally less than an arcsecond with a contrast of 5 to 10 magnitudes (computed as $2.5 \log \left(F_{p} / F_{s}\right)$, where $F$ is the flux and $p$ and $s$ indicate primary and secondary, respectively).

In some situations, direct images can actually resolve the primary. A 50-100 km diameter asteroid at 2 au subtends 34-68 mas while the diffraction limit of a $10 \mathrm{~m}$ telescope at a typical imaging wavelength of $1.2 \mu \mathrm{m}$ is about 30 mas. Although the diffraction limit is not reached, it can be approached with high-performance AO instruments in excellent conditions. With a sequence of disk-resolved images that provide sufficient orientational coverage, it is possible to estimate the 3D shape of the primary. This enables volume and density determinations.

Instruments capable of meeting the contrast and resolution requirements include the Hubble Space Telescope (HST) and large (10 m class) ground-based telescopes equipped with AO. Spacecraft encounters provide an opportunity to detect small satellites at small separations because of proximity to the target and the absence of the point spread function halo that affects ground-based AO instruments.

At the time Asteroids III was published, MBA satellite discoveries included one by spacecraft ((243) Ida), one by HST ((107) Camilla), and 6 by ground-based AO instruments. Since then, ground-based AO instruments have been responsible for almost all large MBA satellite discoveries: (121) Hermione (Merline et al. 2002b), (379) Huenna (Margot 2003), (130) Elektra 


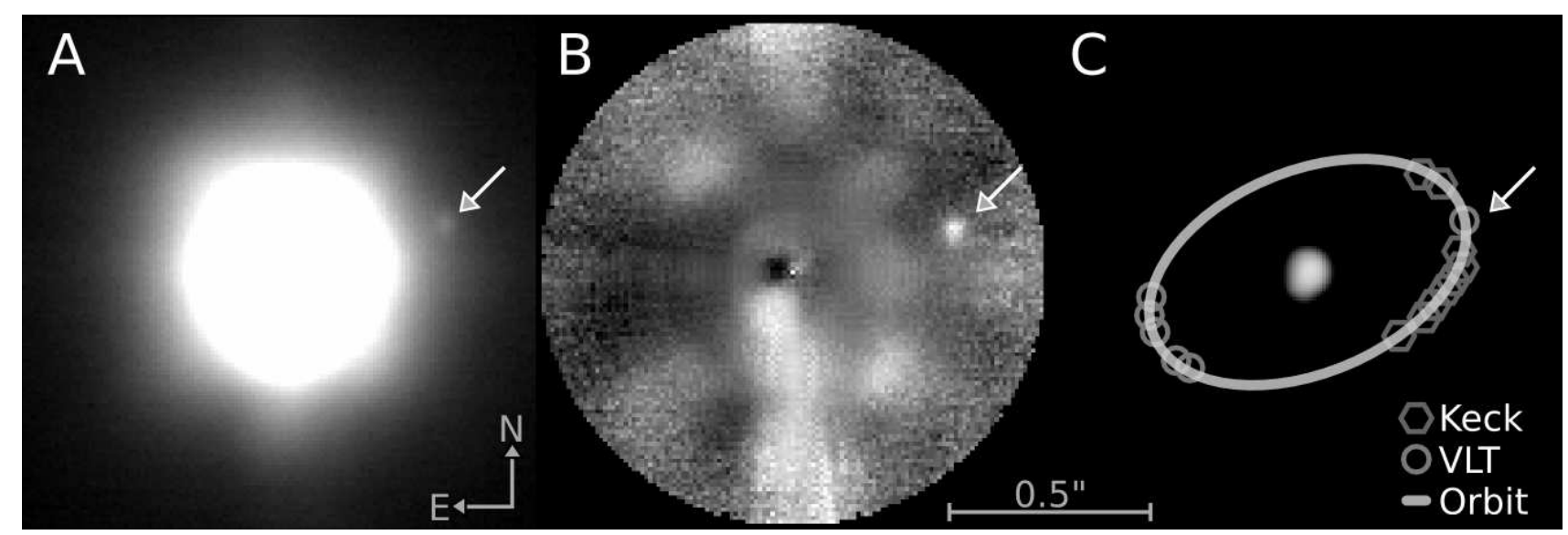

Fig. 6.- Satellite detection by direct imaging with adaptive optics (AO). (a) Image of asteroid (41) Daphne (Vmag=10) obtained with a ground-based AO-fed camera (NACO at ESO VLT, 5 s exposure). (b) Same image after subtraction of the flux from the primary, enabling more accurate measurements of the flux and position of the secondary. (c) Orbit determination. The relative positions of the satellite from VLT/NACO and Keck/NIRC2 images are indicated. Figure adapted from Carry (2009).

(Merline et al. 2003c), a second satellite to (87) Sylvia (Marchis et al. 2005b) and to (45) Eugenia (Marchis et al. 2007), (702) Alauda (Rojo and Margot 2007), (41) Daphne (Conrad et al. 2008), two satellites to (216) Kleopatra (Marchis et al. 2008b) and (93) Minerva (Marchis et al. 2009), and (317) Roxane (Merline et al. 2009). The wide binaries (1509) Esclangona (Merline et al. 2003a) and (4674) Pauling (Merline et al. 2004), which are small asteroids in our classification, have also been identified using AO-fed cameras. HST enabled detections of two additional wide binaries: (22899) $1999 \mathrm{TO}_{14}$ (Merline et al. 2003b) and (17246) $2000 \mathrm{GL}_{74}$ (Tamblyn et al. 2004), both of which are small MBAs. No satellites have been discovered around any of the 7 asteroids recently visited by spacecraft: (4) Vesta, (21) Lutetia, (2867) Šteins, (4179) Toutatis, (5535) Annefrank, (25143) Itokawa, and (132524) APL. The number of known large MBAs with satellites is now 16 , which includes the only known large doubly synchronous system, (90) Antiope (Merline et al. 2000; Michałowski et al. 2004; Descamps et al. 2007, 2009). The fraction of large MBAs with satellites is difficult to estimate because of a complex dependence of satellite detectability on primary-to-secondary angular separation and primary-to-secondary flux ratio. However, because several independent programs have sur- veyed over 300 large MBAs, it is likely that the abundance of binaries in large MBAs is substantially smaller than the $\sim 16 \%$ abundance in NEAs and small MBAs.

Properties of large MBA binaries and triples are summarized in Figs. 7 and 8. With the exception of the nearly equal-mass binary (90) Antiope, the known satellites have secondary-to-primary mass ratios between $10^{-6}$ and $10^{-2}$. All have orbital periods between 1 and 5.5 days, except (379) Huenna, whose orbit has a period of $\sim 88$ days and an eccentricity of $\sim 0.2$ (Marchis et al. 2008c). Many orbits have near-zero eccentricity (e.g., Marchis et al. 2008a), likely the result of tidal damping, but the inner satellites of triples generally have non-zero eccentricities. These eccentricities may have originated when orbits crossed mean motion resonances while tidally expanding (e.g., Fang et al. 2012).

At first glance, large MBA densities appear to cluster in two groups, between 1 and $2 \mathrm{~g} / \mathrm{cm}^{3}$ and above $3 \mathrm{~g} / \mathrm{cm}^{3}$. However, interpretations are limited by the possibility of systematic errors, including overestimates of volumes and underestimates of densities (Pravec and Harris 2007). Because volume uncertainties almost always dominate the error budget for binary asteroid densities (e.g., Merline et al. 2002c; Carry 2012), it is important to assess the realism of uncertainties 

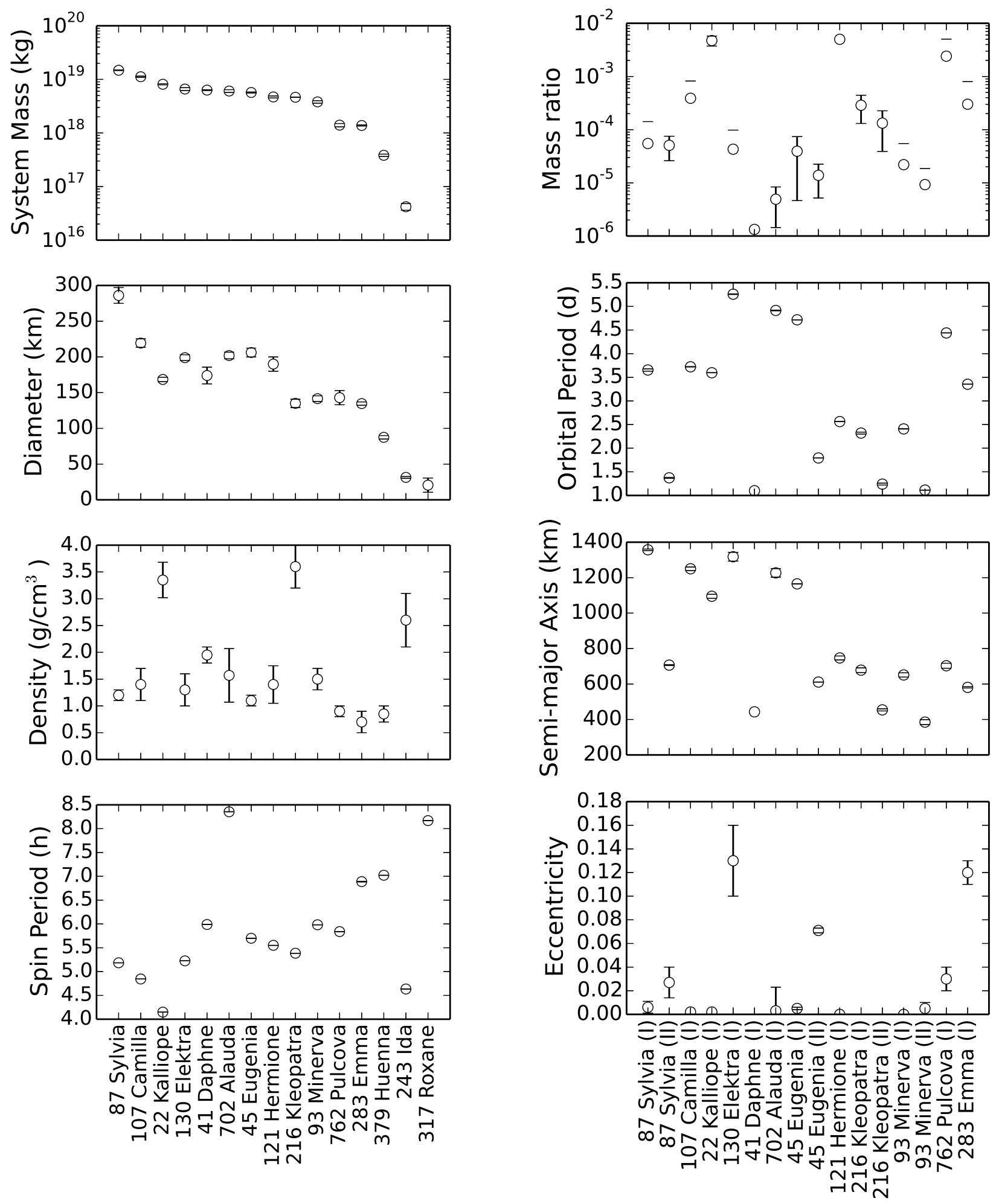

Fig. 7.- Properties of large MBA binaries and triples, excluding the doubly synchronous (90) Antiope. Error bars or upper limits, when available, are shown. Figures 7 and 8 are based on data compiled by Johnston (2014) from references therein.

Fig. 8. - Properties of satellites of large MBAs, excluding outliers (90) Antiope and (379) Huenna (see text). Satellites of (243) Ida and (317) Roxane, whose orbits are not well known, are not shown. 
associated with volume determinations. Some published density values should be regarded with caution because overconfidence in the fractional uncertainty of volume estimates has led to underestimates of bulk density uncertainties. The platinum standard of an orbiting spacecraft yields densities with $\sim 1 \%$ accuracy. The gold standard of radar observations where tens of images with hundreds or thousands of pixels per image are used to reconstruct a detailed 3D shape model yields volumes (and densities) with $\sim 10 \%$ accuracy. In contrast, AO images contain at most a few independent resolution cells of the target asteroid. Shape reconstructions based on AO images and/or lightcurve data may not routinely yield volume accuracies at the $10 \%$ level, although one analysis reached that level (Carry et al. 2012). In the absence of precise volume information, one might be tempted to infer bulk densities from the theory of fluid equilibrium shapes, but this approach is problematic (Holsapple 2007; Harris et al. 2009).

In the Jupiter trojan population, one satellite to (624) Hektor has been reported (Marchis et al. 2006b) since the discovery of the first trojan satellite to (617) Patroclus (Merline et al. 2001). These are the only trojans confirmed to have satellites in spite of several active search programs. The apparent low abundance of binary trojans is intriguing and, if confirmed, may provide additional support for the idea that Jupiter trojans originated in the trans-Neptunian region (Morbidelli et al. 2005; Levison et al. 2009) where they experienced a different collisional environment than in the main belt of asteroids. (624) Hektor has a satellite in a $\sim 3$-day orbit that is eccentric $(\sim 0.3)$ and inclined $\left(\sim 50^{\circ}\right)$ with respect to Hektor's equator (Marchis et al. 2014). (617) Patroclus is unusual because it has two components of similar size in a relatively tight $(\sim 680 \mathrm{~km})$ orbit, with a normalized total angular momentum exceeding that available from fission of a single parent body (Marchis et al. 2006a).

In the trans-Neptunian region, 14 and 64 binary systems have been discovered with AO and HST, respectively (Johnston 2014). The apparent larger abundance of binary TNOs in the cold classical belt may be due to a different dynamical environment and formation mechanism (Section 5).

Objects in the trojan and TNO populations are generally too faint for AO observations in natural guide star (NGS) mode, in which the science target is also used to measure the properties of the wavefront and command the deformable mirror. These objects can be observed in appulse when their sky position happens to be within $\lesssim 1$ arcminute of a bright star. The advent of laser guide star (LGS) adaptive optics has been an important development that has freed the observer from finding such chance alignments and has opened up a larger fraction of the sky for observation of faint objects. Even with LGS, however, the availability of a tip-tilt star (Rmag $\lesssim 18)$ within $\lesssim 1$ arcminute of the target is still required.

High-resolution and high-contrast imaging capabilities are aggressively sought by instrument builders, in part to enable direct imaging of exoplanets. Cameras equipped with highperformance $\mathrm{AO}$ are currently being installed or commissioned on large ground-based telescopes: HiCIAO on Subaru, GPI on Gemini, and SPHERE at the ESO VLT. These instruments will improve the ability to detect faint satellites orbiting close to their respective primaries. However, in most cases, asteroids fall in the faint-end range of these instrument capabilities. The next generation of large telescopes ( $~ 30$ m diameter) such as the Thirty Meter Telescope (TMT) and European Extremely Large Telescope (E-ELT) will provide an improvement in sensitivity by a factor of $\sim 10$ and in angular resolution by a factor of $\sim 3$ compared to current $10 \mathrm{~m}$ telescopes. With the anticipated development of AO capabilities at shorter wavelengths, the second generation of instruments at these facilities is expected to provide improvements in angular resolution by a factor of $\sim 5$. Such instruments may allow detection of the small MBA binaries that are currently beyond the reach of direct imaging instruments. In many of these systems, the components are separated by only a few mas and the size ratios are larger than in large MBA binaries, resulting in flux ratios closer to unity. 


\subsection{Spectral Observations of MBA and Trojan Systems}

It is generally difficult to separate the light emitted or reflected from the secondary from that of the brighter primary. Nevertheless, such observations can be attempted when the secondary happens to be at a large angular separation from the primary, when the system is undergoing mutual events, or with the help of an integral field spectrograph.

Spectra of (22) Kalliope and its satellite Linus in the 1-2.4 $\mu \mathrm{m}$ region appear to be similar (Laver et al. 2009), which the authors attribute to satellite formation after a major impact on the precursor body. Observations of both components of (90) Antiope in the same spectral region also shows surface reflectances that are similar (Marchis et al. 2011). The spectrum of (379) Huenna is characteristic of C-type asteroids and the secondary does not exhibit a significantly different taxonomic type (DeMeo et al. 2011). Both components of (809) Lundia are consistent with a V-type classification (Birlan et al. 2014).

In the mid-infrared, Spitzer observations of the trojan (617) Patroclus, including during mutual events, provided size estimates for its components and a thermal inertia of $20 \pm 15 \mathrm{~J} \mathrm{~s}^{-1 / 2} \mathrm{~K}^{-1} \mathrm{~m}^{-2}$ (Mueller et al. 2010). Spitzer observations combined with photometric results in the visible yielded size and albedo estimates for (624) Hektor (Emery et al. 2006). Spitzer observations of these and other binaries did not resolve the binaries and results typically cannot be compared to observations that place many resolution elements on individual components. One exception is $2000 \mathrm{DP}_{107}$, where analysis of Spitzer data yields a system density of $0.9 \pm 0.3 \mathrm{~g} / \mathrm{cm}^{3}$ (Marchis et al. 2012) and the radar results indicate $1.4 \pm$ $0.2 \mathrm{~g} / \mathrm{cm}^{3}$ (Naidu et al. 2015).

\subsection{Stellar Occultations of MBA and Trojan Systems}

Stellar occultations provide a way of detecting components of a multiple-asteroid system, of placing bounds on component sizes, and of obtaining the relative positions of components on the plane of the sky. A recording of star light as a function of time shows a deep extinction when a target body crosses the line of sight between the observer and the star. This can be interpreted in terms of a chord on the apparent disk of the target body projected on the plane of the sky. If several observers are placed across the occultation path on the surface of the Earth, multiple chords can be obtained, and the size and shape of the target projected on the sky can be reconstructed (Fig. 9). When two or more components are present, it is also possible to measure their relative position. While the reliability of this technique was disputed a decade ago due to the lack of digital recordings, the availability of low-cost cameras and global positioning systems has enabled a dramatic improvement in the precision of timing reports. Stellar occultations have become an important observational tool for the study of binary asteroids.

Early reports (e.g., Binzel and van Flandern 1979) of asteroids suspected to be binaries on the basis of occultation data (including (3) Juno, (6) Hebe, (9) Metis, (12) Victoria, (18) Melpomene, (146) Lucina, and (532) Herculina) have remained largely unconfirmed despite extensive follow-up searches. However, it is likely that the outer satellite of (216) Kleopatra was detected during a 1980 occultation (Dunham 1981; Descamps et al. 2011). The detection of a satellite around the trojan (911) Agamemnon has been suggested (Timerson et al. 2013) but not yet confirmed. The occultation technique has also been used to detect rings around the centaur (10199) Chariklo (Braga-Ribas et al. 2014).

One strength of the stellar occultation technique lies in the fact that the observability of the event depends mainly on the brightness of the star and not of the asteroid or satellite. Stellar occultations can thus be used to detect small ( $\mathrm{km}$ size) satellites, even those that are close to the primary and that would remain undetected in direct imaging.

Another strength of the technique is the potential for high-precision measurements. Stellar occultations are based on time-series photometry. Given a sufficiently high cadence (e.g., 10-30 im- 
ages per second), it is possible to obtain a precision of a few mas on the relative position of binary components, which is 5 to 10 times better than with direct imaging with current instrumentation.

Finally, well-sampled stellar occultations allow for recovery of the size and apparent shape of asteroids and their satellites, whereas optical lightcurves and direct imaging observations provide primarily the diameter ratio of the components and more limited shape information. So far, four successful observations of satellite size and shape have been reported: Linus, satellite of (22) Kalliope (Descamps et al. 2008), Romulus, the outer satellite of (87) Sylvia (Berthier et al. 2014), and both components of the equal-size binaries (90) Antiope (Bartczak et al. 2014) and (617) Patroclus (Buie et al. 2014).

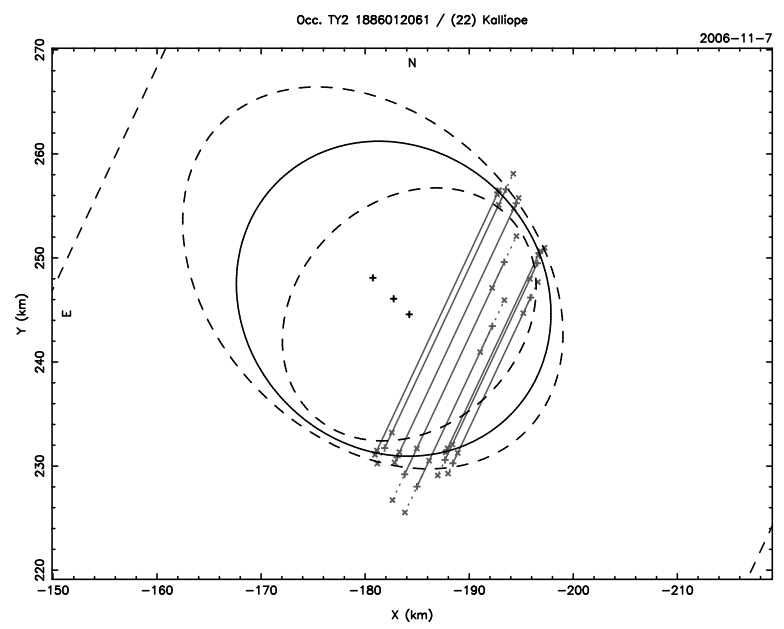

Fig. 9.- The apparent shape of Linus, a satellite of (22) Kalliope (Margot and Brown 2003), detected by stellar occultations. In this analysis, the profile of the satellite (solid curve) fitted to the observed chords (straight lines) yields an equivalent diameter of $30 \pm$ $6 \mathrm{~km}$. Dashed curves show the corresponding uncertainty of the fitted profile, and dashed lines show negative detections. Figure adapted from Descamps et al. (2008).

Despite all of these strengths, there remains a relatively low number of well-covered stellar occultation events. This is due, in part, to the requirement of successful observations at many stations. Owing to uncertainties on both the star and asteroid positions, the occultation path can shift by several tens or even hundreds of $\mathrm{km}$ on Earth compared to the prediction. Observers must therefore spread geographically to cover an event, but the detection of a satellite by several stations requires a fine grid of observers.

The situation is, however, expected to improve dramatically with the availability of the Gaia stellar catalog and better asteroid orbits (Tanga and Delbo 2007). Predictions of the occultation paths (for the center of mass) will be accurate to a few $\mathrm{km}$, and the main source of uncertainty will become the prediction of the relative position of the satellite around the primary.

\subsection{Other Observations}

There have been several attempts to use groundbased interferometers to measure the angular separation of binary systems (Delbo et al. 2009; Carry et al. 2015). However, asteroid satellites are too faint for current interferometers operating in the visible and near-infrared and at the edge of detection in the mid-infrared. Future instrumentation may allow such observations. There are also prospects for observations with the ALMA sub-millimeter array (Busch 2009).

\section{DYNAMICS}

In parallel with advances in instrumentation and observing capabilities, the field has seen tremendous developments in understanding the dynamical processes that affect asteroid systems. This has been enabled in large part by the availability of detailed shape models and orbital parameters, by the need to model the dynamics of newly discovered triple systems, and by the desire to understand formation and evolution processes.

A non-exhaustive list of some dynamical problems that have been explored since Asteroids III includes the stability of asteroid satellite orbits (Scheeres 2002; Frouard and Compère 2012), the dynamics around triaxial bodies (Scheeres 2009a), the fate of asteroid ejecta (Scheeres 2007), the formation of contact binaries via dynamical evolution (Scheeres 2009a; Taylor and Margot 2011, 2014), the genesis of eccentric and mutually inclined orbits (Fang et al. 2011; Fang 
and Margot 2012c), the orbital determination of triple systems using point-mass approximations (Marchis et al. 2010) and full N-body calculations (Fang et al. 2012), the influence of Kozai cycles on binaries (Perets and Naoz 2009; Fang and Margot 2012b), the effects of close planetary encounters on mutual orbits (Fang and Margot 2012a) and spin states (Takahashi et al. 2013), the complex spin-orbit interactions with irregular component shapes (Scheeres et al. 2006), including the libration and irregular rotation of secondaries (Naidu and Margot 2015), the influence of internal structure (Goldreich and Sari 2009), material properties (Taylor and Margot 2011) and nonspherical shapes (Taylor and Margot 2014) on tidal evolution, the possibility of tidal saltation (Harris et al. 2009; Fahnestock and Scheeres 2009), the possibility of significant radiative evolution (Ćuk and Burns 2005; Ćuk 2007; Ćuk and Nesvorný 2010; McMahon and Scheeres 2010a,b), and the possibility of a stable equilibrium between tidal and radiative evolution (Jacobson and Scheeres 2011a).

Several radar data sets provide exquisite constraints for dynamical studies. Reflex motion has been measured for $2000 \mathrm{DP}_{107}$ (Margot et al. 2002; Naidu et al. 2015), $1999 \mathrm{KW}_{4}$ (Ostro et al. 2006), and $1991 \mathrm{VH}$ (Naidu et al. 2012), allowing masses of individual components to be determined. Because detailed component shapes are also available, one can fully model the system dynamics and study spin-orbit coupling in detail (Scheeres et al. 2006; Fahnestock and Scheeres 2008; Naidu and Margot 2015). One finding from this work is that even moderately elongated secondaries on mildly eccentric orbits are likely to experience chaotic rotation that substantially affect binary evolution timescales (Fig. 10).

\section{SMALL ASTEROIDS: SYNTHESIS}

\subsection{Rotational Fission Hypothesis}

With the exception of the doubly synchronous binary asteroid systems, the primary asteroids of all small binary systems are rapidly rotating

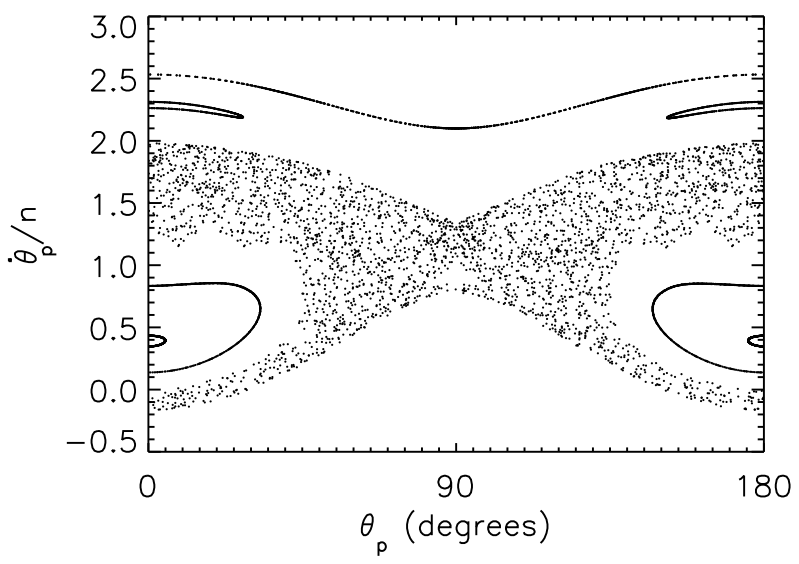

Fig. 10. - Surface of section plot showing the possible rotational regimes of the $\sim 200 \mathrm{~m}$ secondary of $1991 \mathrm{VH}$ (secondary elongation $a / b=1.5$ and mutual orbit eccentricity $e=0.05$ ). The plot shows the angle between the long axis and the line of apsides of the mutual orbit, $\theta_{p}$, against its time derivative, $\dot{\theta_{p}}$, normalized by the mean motion, $n$, at each pericenter passage. Five trajectories are illustrated (from top to bottom: non-resonant quasi-periodic, periodic, chaotic, periodic, periodic). While trapped in the sea of chaos, the secondary experiences torques on its permanent deformation that result in a highly variable spin rate, preventing BYORP-type evolution. Figure from Naidu and Margot (2015).

(within a factor of only a few of the critical disruption spin limit for bodies with no shear or tensile strength $\omega_{d}=\sqrt{4 \pi \rho G / 3}$ ). Furthermore, almost all known small binary asteroids have high angular momentum contents (Pravec and Harris 2007). These characteristics are not consistent with formation following a sub-catastrophic impact, capture through a three-body interaction in the near-Earth or main belt, or capture after a catastrophic impact. Instead, they are indicative of formation from a rotational fission event (e.g., Margot et al. 2002; Pravec and Harris 2007). The rotational fission hypothesis posits that a parent asteroid can be torqued to a rotation rate so great that the centrifugal accelerations overpower the gravitational accelerations holding a strengthless asteroid together (Weidenschilling 1980). It is possible that some small asteroids have cohesive or molecular strength in addition 
to self-gravity (e.g., Rozitis et al. 2014). In these cases, the centrifugal accelerations must overcome these additional forces in order for the asteroid to fission (Pravec and Harris 2000; Sánchez and Scheeres 2014). At rapid rotation rates, loose surface material can flow from high-latitude regions to the equator along potential gradients (Ostro et al. 2006). It has been shown that rotational acceleration could trigger local slope failures and landslides, which can form the canonical top shape and equatorial bulge seen on primary components in small multiple-asteroid systems (Walsh et al. 2008; Harris et al. 2009).

Bottke et al. (2002) proposed a YORP-induced rotational fission hypothesis. It has since been shown that the YORP effect controls the rotational acceleration of small asteroids (Bottke et al. 2006; Marzari et al. 2011) and naturally explains the period distribution among small asteroids (Pravec et al. 2008; Rossi et al. 2009; Polishook and Brosch 2009). Furthermore, including the YORP-induced rotational fission hypothesis in size-frequency distribution models improves the agreement with observations (Jacobson et al. 2014a). The observed characteristics of the systems described in Sections 2.1-2.3 as well as thermal inertia observations (Delbo et al. 2011) are consistent with a binary formation mechanism that involves spin-up and mass shedding. The YORP-induced rotational fission hypothesis is the leading candidate for explaining the formation of binaries, triples, and pairs among small asteroids.

\subsection{Asteroid Pairs}

The YORP effect can increase the spin rate of asteroids beyond the critical disruption spin limit, thereby triggering rotational fission. In actuality, there is some uncertainty regarding the spin rate at which disruption occurs - there may be failure and deformation before fission (Walsh et al. 2008; Sánchez and Scheeres 2011; Cotto-Figueroa et al. 2013). The critical disruption spin limit also depends on the detailed shapes, masses, interlocking nature of the interior components and any cohesive forces (Scheeres 2007, 2009b; Sánchez and Scheeres 2014). Despite ignoring these details, simple calculations provide a rotational fis- sion model that can be compared directly and successfully with observations.

If a spherical approximation of each component is made, then the rotational breakup spin rate $\omega_{q}$ necessary for fission as a function of the secondary-to-primary mass ratio $q$ is (Scheeres 2007):

$$
\omega_{q}=\omega_{d} \sqrt{\frac{1+q}{\left(1+q^{1 / 3}\right)^{3}}} .
$$

This is the exact solution for two spheres resting on each other with a mass ratio of $q$ and rotating about the axis of maximum moment of inertia.

The spherical component model described above demonstrates the important reality that the larger the mass ratio $q$ of the two future binary members, the slower the required rotation rate necessary to create the binary system. This slower required rotation rate translates into a small initial free energy for the ensuing binary system. The free energy $E_{f}$ is the energy that is accessible to the different energy reservoirs in the system, including the rotation states of each member and the orbit. It does not include the internal binding energy of each object. The free energy is an important quantity because it determines the boundedness of the system. Bound systems have negative free energy, while unbound systems have positive free energy. An unbound binary system implies that the system is capable of disruption but does not imply that the system will disrupt. For the idealized case of two spheres, the free energy can be expressed as (Scheeres 2007):

$$
E_{f}=\frac{2 \pi \rho \omega_{d}^{2} R_{p}^{5}}{15} f(q),
$$

where $R_{p}$ is the radius of the primary and $f(q)$ is an algebraic, monotonically decreasing function for $0<q \leq 1$. For the equation above corresponding to two spheres, the function crosses zero when $q \approx 0.204$. Similar equations can be written for any two component shapes, but $q \sim 0.2$ remains near the binding energy transition point, so the model uses this point as a simple approximation. This crossing point divides bound systems with negative energy and mass ratios $q>0.2$ and unbound systems with positive energy and 
mass ratios $q<0.2$. Because of this fundamental difference, high mass ratio $q>0.2$ and low mass ratio $q<0.2$ binary systems evolve differently (Scheeres 2009a; Jacobson and Scheeres 2011b). Primarily, positive energy low mass ratio systems will chaotically explore orbital phase space until the majority find a disruption trajectory creating an asteroid pair; this evolutionary route is unavailable to high mass ratio systems.

The asteroid pair population provides a natural laboratory to test this relationship (Scheeres 2007; Vokrouhlický and Nesvorný 2008). Pravec et al. (2010) examined many asteroid pair systems and measured the rotation rate of the primary and the absolute magnitude difference between the pair members. These two quantities should follow a simple relationship related to $\omega_{q}$, although many of the ignored details mentioned at the beginning of this section can move asteroids away from this relationship. Indeed, Pravec et al. (2010) discovered that asteroid pairs do follow this relationship (Fig. 5). Furthermore, they found that the large members of asteroid pairs have a broader range of elongations than the primaries of binary systems, consistent with the findings of Jacobson and Scheeres (2011b) that prolate primaries are less likely to remain in a bound binary system after rotational fission. Thus, there is strong evidence to support the hypothesis that asteroid pairs are the products of rotational fission.

Asteroid pairs continue to be a fertile observational landscape. Since dynamical integrations can derive the "birthdate" of such systems, observers can test ideas regarding space weathering timescales and YORP evolution after fission (Polishook et al. 2014a; Polishook 2014). Along with binary systems, the surfaces of asteroid pairs may provide clues in the future regarding the violence of the rotational fission process (Polishook et al. 2014b).

\subsection{Binary and Triple Systems}

Jacobson and Scheeres (2011b) showed that after rotational fission there are a number of possible outcomes. Their numerical studies produced the evolutionary flow chart shown in Fig. 11; many of these outcomes were also found by Fang and Margot (2012c). The high and low mass ratio distinction for rotational fission emphasized above plays an important role in distinguishing the two evolutionary pathways. Along the high mass ratio pathway, both binary members tidally synchronize and then evolve according to the BYORP effect.

Along the low mass ratio pathway, the binary system is unbound. Since these systems are chaotic, many are disrupted and become asteroid pairs. During this chaotic binary state, the secondary can often go through rotational fission itself, although this rotational fission is torqued by spin-orbit coupling (Fig. 10) rather than the YORP effect. Loss of material from the secondary stabilizes the remaining orbiting components. The lost mass may reaccrete onto the primary, perhaps contributing to the observed equatorial ridges, or may escape from the system. In these cases, the system undergoes another chaotic binary episode with three possible outcomes: a re-shaped asteroid, an asteroid pair, or a stable binary. These binaries still possess positive free energy such that they may disrupt if disturbed. In other cases, the system retains three components after secondary fission. While the numerical simulations of Jacobson and Scheeres (2011b) did not yield this latter outcome, it is possible that this pathway explains the existence of stable triple systems.

After stabilization of the low mass ratio binary system, the secondary synchronizes due to tides (e.g., Goldreich and Sari 2009), although some satellites may be trapped in a chaotic rotation state for durations that exceed the classic spin synchronization timescales (Naidu and Margot 2015). Then the system evolves according to the BYORP effect and tides. These binary evolutionary processes and their outcomes are discussed in Walsh \& Jacobson (this volume). As shown in Fig. 11, these evolutionary paths include each of the binary morphologies identified in this chapter and by other teams (Pravec and Harris 2007; Fang and Margot 2012c). In particular, the formation of wide asynchronous binaries such as (1509) Esclangona, (4674) Pauling, (17246) 2000 


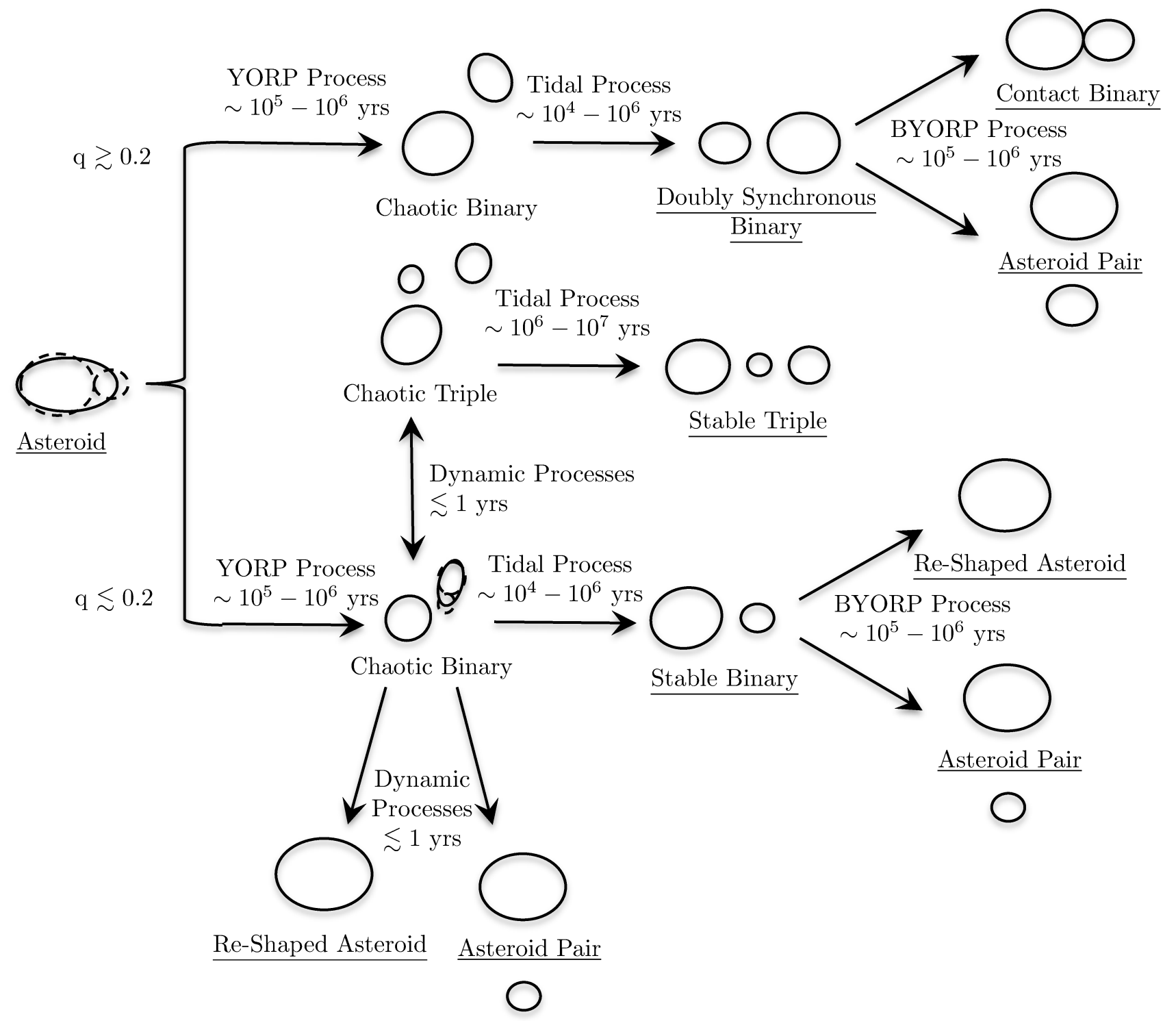

Fig. 11.- Flowchart showing the possible evolutionary paths for an asteroid after it undergoes rotational fission. Each arrow is labeled with the dominant process and an estimated timescale for this process. Underlined states are nominally stable for a YORP effect timescale. Figure from Jacobson and Scheeres (2011b).

$\mathrm{GL}_{74}$, and (22899) $1999 \mathrm{TO}_{14}$ is best explained by a rotational fission mechanism (Polishook et al. 2011) followed by BYORP orbital expansion (Jacobson et al. 2014b).

An alternative formation mechanism for triples such as (153591) $2001 \mathrm{SN}_{263}$ and (136617) 1994 $\mathrm{CC}$ is that after creating a stable binary system, the primary undergoes rotational fission a second time. As long as the third component is on a distant enough orbit, then this process may result in a stable triple system (Fang et al. 2011; Fang and Margot 2012c; Jacobson et al. 2014b).

\section{LARGE ASTEROIDS: SYNTHESIS}

The primaries of most known binary and triple asteroids greater than $20 \mathrm{~km}$ have spin periods in the range of $4 \mathrm{~h}$ to $7 \mathrm{~h}$ (Fig. 7). While these spin rates are not near the disruption spin limit, they are typically faster than the mean spin rates for asteroids of similar sizes. The total angular momentum content, however, is well below that required for rotational fission. The secondary-to-primary mass ratios in these systems range from $10^{-6}$ to $10^{-2}$. These properties are consistent with satel- 
lite formation during large collisions (Fig. 12). Durda et al. (2004) have shown in numerical simulations that impacts of $10-$ to $30-\mathrm{km}$ diameter projectiles striking at impact velocities between $3 \mathrm{kms}^{-1}$ and $7 \mathrm{kms}^{-1}$ can produce satellites that match observed properties. Multiple asteroid systems, e.g., (45) Eugenia (Merline et al. 1999; Marchis et al. 2007) and (87) Sylvia (Margot and Brown 2001; Marchis et al. 2005a) can also plausibly form through collisions.

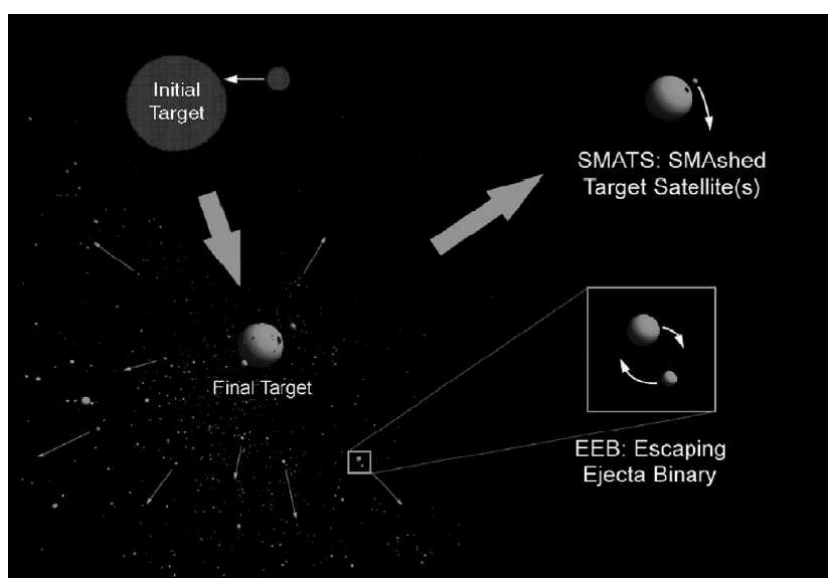

Fig. 12.- Numerical simulations show that binaries can form as a result of large impacts between asteroids. In some scenarios, impact debris can remain gravitationally bound to the target body, forming a satellite (SMATs). This process likely explains the formation of large MBA binaries. In other scenarios, two fragments from the escaping ejecta have sufficiently similar trajectories, such that they become bound to one another (EEBs). Figure from Durda et al. (2004).

There is more uncertainty related to the formation of (90) Antiope and (617) Patroclus, which are both too large to be substantially affected by YORP. Hypotheses for the formation of (90) Antiope include primordial fission due to excessive angular momentum (Pravec and Harris 2007), an improbable low-velocity collision of a large impactor (Weidenschilling et al. 2001), or shrinking of an initially wide binary formed by gravitational collapse (Nesvorný et al. 2010). Gravitational collapse in a gas-rich protoplanetary disk has been invoked to explain the formation of numerous binaries in the trans-Neptunian region. (617) Patroclus may be a primordial TNO that avoided disruption during emplacement in the trojan re- gion (Nesvorný et al. 2010). Wide TNO binaries would not be expected to survive this process, whereas encounter calculations (e.g., Fang and Margot 2012a) show that tight binaries would.

\section{CONCLUSIONS}

Studies of binaries, triples, and pairs remain a fertile ground for observing processes that are important in planet formation and for measuring quantities that are difficult to obtain by other means. These include masses and densities as well as thermal, mechanical, and interior properties. Binaries or triples have been found in $\sim 50$ NEAs, 50 small MBAs, 20 large MBAs, and 2 trojans. A unifying paradigm based on rotational fission and post-fission dynamics explains the formation of small binaries, triples, and pairs. Because the sun-powered rotational fission process is unrelenting, and because the production of pairs is a frequent outcome of this process, a substantial fraction of small bodies likely originated in a rotational disruption event. This origin affects the size distribution of asteroids and may explain the presence of single NEAs with equatorial bulges observed with radar. Small satellites of large MBAs are likely formed during large collisions. Advances in instrumentation, observational programs, and analysis techniques hold the promise of exciting findings in the next decade.

\section{REFERENCES}

P. Bartczak, T. Michałowski, T. Santana-Ros, and G. Dudziński. A new non-convex model of the binary asteroid 90 Antiope obtained with the SAGE modelling technique. Mon. Not. R. Astron. Soc., 443:1802-1809, 2014.

T. M. Becker, E. S. Howell, M. C. Nolan, C. Magri, P. Pravec, P. A. Taylor, J. Oey, D. Higgins, J. Világi, L. Kornoš, A. Galád, Š. Gajdoš, N. M. Gaftonyuk, Y. N. Krugly, I. E. Molotov, M. D. Hicks, A. Carbognani, B. D. Warner, F. Vachier, F. Marchis, and J. T. Pollock. Physical modeling of triple near-Earth Asteroid (153591) $2001 \mathrm{SN}_{263}$ from radar and optical 
light curve observations. Icarus, 248:499-515, 2015.

R. Behrend, L. Bernasconi, R. Roy, A. Klotz, F. Colas, P. Antonini, R. Aoun, K. Augustesen, E. Barbotin, N. Berger, H. Berrouachdi, E. Brochard, A. Cazenave, C. Cavadore, J. Coloma, V. Cotrez, S. Deconihout, C. Demeautis, J. Dorseuil, G. Dubos, R. Durkee, E. Frappa, F. Hormuth, T. Itkonen, C. Jacques, L. Kurtze, A. Laffont, M. Lavayssière, J. Lecacheux, A. Leroy, F. Manzini, G. Masi, D. Matter, R. Michelsen, J. Nomen, A. Oksanen, P. Pääkkönen, A. Peyrot, E. Pimentel, D. Pray, C. Rinner, S. Sanchez, K. Sonnenberg, S. Sposetti, D. Starkey, R. Stoss, J.-P. Teng, M. Vignand, and N. Waelchli. Four new binary minor planets: (854) Frostia, (1089) Tama, (1313) Berna, (4492) Debussy. Astron. Astrophys., 446:1177-1184, February 2006.

M. Belton, C. Chapman, P. Thomas, M. Davies, R. Greenberg, K. Klaasen, D. Byrnes, L. D'Amario, S. Synnott, W. Merline, J.-M. Petit, A. Storrs, and B. Zellner. The bulk density of asteroid 243 Ida from Dactyl's orbit. Nature, 374:785-788, 1995.

L. A. M. Benner, J. L. Margot, M. C. Nolan, J. D. Giorgini, M. Brozovic, D. J. Scheeres, C. Magri, and S. J. Ostro. Radar Imaging and a Physical Model of Binary Asteroid 65803 Didymos. In Bull. Am. Astron. Soc., volume 42, 2010.

J. Berthier, F. Vachier, F. Marchis, J. Ďurech, and B. Carry. Physical and dynamical properties of the main belt triple Asteroid (87) Sylvia. Icarus, 239:118-130, 2014.

R. P. Binzel and T. C. van Flandern. Minor planets - The discovery of minor satellites. Science, 203:903-905, 1979.

M. Birlan, O. Vaduvescu, A. Tudorica, A. Sonka, A. Nedelcu, A. Galad, F. Colas, F. Pozo N., A. Barr D., R. Toma, I. Comsa, P. Rocher, V. Lainey, D. Vidican, D. Asher, C. Opriseanu, C. Vancea, J. P. Colque, C. P. Soto, R. Rekola, and E. Unda-Sanzana. More than 160 near Earth asteroids observed in the EURONEAR network. Astron. Astrophys., 511:A40, 2010.

M. Birlan, D. A. Nedelcu, M. Popescu, P. Vernazza, F. Colas, and A. Kryszczyńska. Spectroscopy and surface properties of (809) Lundia. Mon. Not. R. Astron. Soc., 437:176-184, 2014.

W. F. Bottke and H. J. Melosh. The formation of asteroid satellites and doublet craters by planetary tidal forces. Nature, 381:51-53, 1996a.

W. F. Bottke and H. J. Melosh. Binary asteroids and the formation of doublet craters. Icarus, 124:372-391, 1996b.

W. F. Bottke, D. Vokrouhlický, D. P. Rubincam, and M. Brož. The Effect of Yarkovsky Thermal Forces on the Dynamical Evolution of Asteroids and Meteorids. In W. F. Bottke, A. Cellino, P. Paolicchi, and R. P. Binzel, editors, Asteroids III, pages 395-408. Univ. of Arizona Press, 2002.

W. F. Bottke, David Vokrouhlický, David P Rubincam, and David Nesvorný. The Yarkovsky and Yorp Effects: Implications for Asteroid Dynamics. Annual Review of Earth and Planetary Sciences, 34:157-191, 2006.

F. Braga-Ribas, B. Sicardy, J. L. Ortiz, C. Snodgrass, F. Roques, R. Vieira-Martins, J. I. B. Camargo, M. Assafin, R. Duffard, E. Jehin, J. Pollock, R. Leiva, M. Emilio, D. I. Machado, C. Colazo, E. Lellouch, J. Skottfelt, M. Gillon, N. Ligier, L. Maquet, G. BenedettiRossi, A. R. Gomes, P. Kervella, H. Monteiro, R. Sfair, M. El Moutamid, G. Tancredi, J. Spagnotto, A. Maury, N. Morales, R. GilHutton, S. Roland, A. Ceretta, S.-H. Gu, X.B. Wang, K. Harpsøe, M. Rabus, J. Manfroid, C. Opitom, L. Vanzi, L. Mehret, L. Lorenzini, E. M. Schneiter, R. Melia, J. Lecacheux, F. Colas, F. Vachier, T. Widemann, L. Almenares, R. G. Sandness, F. Char, V. Perez, P. Lemos, N. Martinez, U. G. Jørgensen, M. Dominik, F. Roig, D. E. Reichart, A. P. 
Lacluyze, J. B. Haislip, K. M. Ivarsen, J. P. Moore, N. R. Frank, and D. G. Lambas. A ring system detected around the Centaur (10199) Chariklo. Nature, 508:72-75, 2014.

H. E. Brooks. Orbits of Binary Near-Earth Asteroids from Radar Observations. In Bull. Am. Astron. Soc., volume 38, 2006.

M. Brozović, L. A. M. Benner, P. A. Taylor, M. C. Nolan, E. S. Howell, C. Magri, D. J. Scheeres, J. D. Giorgini, J. T. Pollock, P. Pravec, A. Galád, J. Fang, J. L. Margot, M. W. Busch, M. K. Shepard, D. E. Reichart, K. M. Ivarsen, J. B. Haislip, A. P. Lacluyze, J. Jao, M. A. Slade, K. J. Lawrence, and M. D. Hicks. Radar and optical observations and physical modeling of triple near-Earth Asteroid (136617) 1994 CC. Icarus, 216:241-256, 2011.

M. W. Buie, C. B. Olkin, W. J. Merline, B. Timerson, D. Herald, W. M. Owen, H. B. Abramson, K. J. Abramson, D. C. Breit, D. B. Caton, S. J. Conard, M. A. Croom, R. W. Dunford, J. A. Dunford, D. W. Dunham, C. K. Ellington, Y. Liu, P. D. Maley, A. M. Olsen, R. Royer, A. E. Scheck, C. Sherrod, L. Sherrod, T. J. Swift, L. W. Taylor, and R. Venable. Shape and Size of Patroclus and Menoetius from a Stellar Occultation. In Bull. Am. Astron. Soc., volume 46, 2014.

M. W. Busch. ALMA and asteroid science. Icarus, 200:347-349, 2009.

M. W. Busch, S. J. Ostro, L. A. M. Benner, M. Brozovic, J. D. Giorgini, J. S. Jao, D. J. Scheeres, C. Magri, M. C. Nolan, E. S. Howell, P. A. Taylor, J. L. Margot, and W. Brisken. Radar observations and the shape of nearEarth asteroid $2008 \mathrm{EV}_{5}$. Icarus, 212:649660, 2011.

B. Carry. Asteroids physical properties from high angular-resolution imaging. $\mathrm{PhD}$ thesis, $\mathrm{Ob}-$ servatoire de Paris, 2009.

B. Carry. Density of asteroids. Planet. Space Sci., 73:98-118, 2012.
B. Carry, M. Kaasalainen, W. J. Merline, T. G. Müller, L. Jorda, J. D. Drummond, J. Berthier, L. O'Rourke, J. Ďurech, M. Küppers, A. Conrad, P. Tamblyn, C. Dumas, H. Sierks, and Osiris Team. Shape modeling technique KOALA validated by ESA Rosetta at (21) Lutetia. Planet. Space Sci., 66:200-212, 2012.

B. Carry, A. Matter, P. Scheirich, P. Pravec, L. Molnar, S. Mottola, A. Carbognani, E. Jehin, A. Marciniak, R. P. Binzel, F. E. DeMeo, M. Birlan, M. Delbo, E. Barbotin, R. Behrend, M. Bonnardeau, F. Colas, P. Farissier, M. Fauvaud, S. Fauvaud, C. Gillier, M. Gillon, S. Hellmich, R. Hirsch, A. Leroy, J. Manfroid, J. Montier, E. Morelle, F. Richard, K. Sobkowiak, J. Strajnic, and F. Vachier. The small binary asteroid (939) Isberga. Icarus, 248:516-525, 2015.

A. Cellino, R. Pannunzio, V. Zappala, P. Farinella, and P. Paolicchi. Do we observe light curves of binary asteroids? Astron. Astrophys., 144: 355-362, 1985.

C. R. Chapman, J. Veverka, P. C. Thomas, K. Klaasen, M. J. S. Belton, A. Harch, A. McEwen, T. V. Johnson, P. Helfenstein, M. E. Davies, W. J. Merline, and T. Denk. Discovery and Physical Properties of Dactyl a Satellite of Asteroid 243 Ida. Nature, 374: $783+, 1995$.

S. R. Chesley, D. Farnocchia, M. C. Nolan, D. Vokrouhlický, P. W. Chodas, A. Milani, F. Spoto, B. Rozitis, L. A. M. Benner, W. F. Bottke, M. W. Busch, J. P. Emery, E. S. Howell, D. S. Lauretta, J. L. Margot, and P. A. Taylor. Orbit and bulk density of the OSIRIS-REx target Asteroid (101955) Bennu. Icarus, 235: 5-22, 2014.

A. R. Conrad, W. M. Keck Observatory, W. J. Merline, J. D. Drummond, P. M. Tamblyn, C. Dumas, B. X. Carry, R. D. Campbell, R. W. Goodrich, W. M. Owen, and C. R. Chapman. S/2008 (41) 1. IAU Circ., 8930, 2008. 
D. Cotto-Figueroa, T. S. Statler, D. C. Richardson, and P. Tanga. Killing the YORP Cycle: A Stochastic and Self-Limiting YORP Effect. In Bull. Am. Astron. Soc., volume 45, 2013.

M. Ćuk. Formation and Destruction of Small Binary Asteroids. Astrophys. J. Lett., 659:L57L60, 2007.

M. Ćuk and J. A. Burns. Effects of thermal radiation on the dynamics of binary NEAs. Icarus, 176:418-431, 2005.

M. Ćuk and D. Nesvorný. Orbital evolution of small binary asteroids. Icarus, 207:732-743, 2010.

M. Delbo, S. Ligori, A. Matter, A. Cellino, and J. Berthier. First VLTI-MIDI Direct Determinations of Asteroid Sizes. Astrophys. J., 694: 1228-1236, 2009.

M. Delbo, K. Walsh, M. Mueller, A. W. Harris, and E. S. Howell. The cool surfaces of binary near-Earth asteroids. Icarus, 212:138148, 2011.

F. E. DeMeo, B. Carry, F. Marchis, M. Birlan, R. P. Binzel, S. J. Bus, P. Descamps, A. Nedelcu, M. Busch, and H. Bouy. A spectral comparison of (379) Huenna and its satellite. Icarus, 212:677-681, 2011.

P. Descamps, F. Marchis, T. Michalowski, F. Vachier, F. Colas, J. Berthier, M. Assafin, P. B. Dunckel, M. Polinska, W. Pych, D. Hestroffer, K. P. M. Miller, R. Vieira-Martins, M. Birlan, J.-P. Teng-Chuen-Yu, A. Peyrot, B. Payet, J. Dorseuil, Y. Léonie, and T. Dijoux. Figure of the double Asteroid 90 Antiope from adaptive optics and lightcurve observations. Icarus, 187:482-499, 2007.

P. Descamps, F. Marchis, J. Pollock, J. Berthier, F. Vachier, M. Birlan, M. Kaasalainen, A. W. Harris, M. H. Wong, W. J. Romanishin, E. M. Cooper, K. A. Kettner, P. Wiggins, A. Kryszczynska, M. Polinska, J.-F. Coliac, A. Devyatkin, I. Verestchagina, and D. Gorshanov. New determination of the size and bulk density of the binary Asteroid 22 Kalliope from observations of mutual eclipses. Icarus, 196:578-600, 2008.

P. Descamps, F. Marchis, T. Michalowski, J. Berthier, J. Pollock, P. Wiggins, M. Birlan, F. Colas, F. Vachier, S. Fauvaud, M. Fauvaud, J.-P. Sareyan, F. Pilcher, and D. A. Klinglesmith. A giant crater on 90 Antiope? Icarus, 203:102-111, 2009.

P. Descamps, F. Marchis, J. Berthier, J. P. Emery, G. Duchêne, I. de Pater, M. H. Wong, L. Lim, H. B. Hammel, F. Vachier, P. Wiggins, J.-P. Teng-Chuen-Yu, A. Peyrot, J. Pollock, M. Assafin, R. Vieira-Martins, J. I. B. Camargo, F. Braga-Ribas, and B. Macomber. Triplicity and physical characteristics of Asteroid (216) Kleopatra. Icarus, 211:1022-1033, 2011.

A. Doressoundiram, P. Paolicchi, A. Verlicchi, and A. Cellino. The formation of binary asteroids as outcomes of catastrophic collisions. Planet. Space Sci., 45:757-770, 1997.

S R Duddy, Stephen C Lowry, S D Wolters, A Christou, Paul R Weissman, Simon F Green, and B. Rozitis. Physical and dynamical characterisation of the unbound asteroid pair 7343154634. Astron. Astrophys., 539:A36, 2012.

S. R. Duddy, S. C. Lowry, A. Christou, S. D. Wolters, B. Rozitis, S. F. Green, and P. R. Weissman. Spectroscopic observations of unbound asteroid pairs using the WHT. Mon. Not. R. Astron. Soc., 429:63-74, 2013.

D. W. Dunham. Recently-observed planetary occultations. Occultation Newsletter, International Occultation Timing Association (IOTA), 2:139-143, 1981.

D. D. Durda. The formation of asteroidal satellites in catastrophic collisions. Icarus, 120: 212-219, 1996.

D. D. Durda, W. F. Bottke, B. L. Enke, W. J. Merline, E. Asphaug, D. C. Richardson, and Z. M. Leinhardt. The formation of asteroid satellites 
in large impacts: results from numerical simulations. Icarus, 170:243-257, 2004.

J. P. Emery, D. P. Cruikshank, and J. Van Cleve. Thermal emission spectroscopy $(5.238 \mu \mathrm{m})$ of three Trojan asteroids with the Spitzer Space Telescope: Detection of fine-grained silicates. Icarus, 182:496-512, 2006.

E. G. Fahnestock and D. J. Scheeres. Simulation and analysis of the dynamics of binary nearEarth Asteroid (66391) 1999 KW4. Icarus, 194:410-435, 2008.

E. G. Fahnestock and D. J. Scheeres. Binary asteroid orbit expansion due to continued YORP spin-up of the primary and primary surface particle motion. Icarus, 201:135-152, 2009.

J. Fang and J. L. Margot. Binary Asteroid Encounters with Terrestrial Planets: Timescales and Effects. Astron. J., 143:25, 2012a.

J. Fang and J. L. Margot. The Role of Kozai Cycles in Near-Earth Binary Asteroids. Astron. J., 143:59, 2012b.

J. Fang and J. L. Margot. Near-Earth Binaries and Triples: Origin and Evolution of Spin-Orbital Properties. Astron. J., 143:24, 2012 c.

J. Fang, J. L. Margot, M. Brozovic, M. C. Nolan, L. A. M. Benner, and P. A. Taylor. Orbits of near-Earth asteroid triples $2001 \mathrm{SN}_{263}$ and 1994 CC: Properties, origin, and evolution. Astron. J., 141:154, 2011.

J. Fang, J. L. Margot, and P. Rojo. Orbits, Masses, and Evolution of Main Belt Triple (87) Sylvia. Astron. J., 144:70, 2012.

J. Frouard and A. Compère. Instability zones for satellites of asteroids: The example of the (87) Sylvia system. Icarus, 220:149-161, 2012.

P. Goldreich and R. Sari. Tidal Evolution of Rubble Piles. Astrophys. J., 691:54-60, 2009.

J. Hanuš, J. Ďurech, M. Brož, B. D. Warner, F. Pilcher, R. Stephens, J. Oey, L. Bernasconi,
S. Casulli, R. Behrend, D. Polishook, T. Henych, M. Lehký, F. Yoshida, and T. Ito. A study of asteroid pole-latitude distribution based on an extended set of shape models derived by the lightcurve inversion method. Astron. Astrophys., 530:A134, 2011.

A. W. Harris, E. G. Fahnestock, and P. Pravec. On the shapes and spins of rubble pile asteroids. Icarus, 199:310-318, 2009.

J. L. Hilton. Asteroids Masses and Densities. In W. F. Bottke, A. Cellino, P. Paolicchi, and R. P. Binzel, editors, Asteroids III, pages 103-112. Univ. of Arizona Press, 2002.

K. A. Holsapple. Spin limits of Solar System bodies: From the small fast-rotators to 2003 EL61. Icarus, 187(2):500-509, 2007.

R. S. Hudson. Three-Dimensional Reconstruction of Asteroids from Radar Observations. Remote Sensing Reviews, 8:195-203, 1993.

S. A. Jacobson and D. J. Scheeres. Long-term Stable Equilibria for Synchronous Binary Asteroids. Astrophys. J. Lett., 736:L19, 2011a.

S. A. Jacobson and D. J. Scheeres. Dynamics of rotationally fissioned asteroids: Source of observed small asteroid systems. Icarus, 214(1): 161-178, 2011b.

S. A. Jacobson, F. Marzari, A. Rossi, D. J. Scheeres, and Donald R Davis. Effect of rotational disruption on the size-frequency distribution of the Main Belt asteroid population. Mon. Not. R. Astron. Soc., page L15, 2014a.

S. A. Jacobson, D. J. Scheeres, and J. McMahon. Formation of the Wide Asynchronous Binary Asteroid Population. Astrophys. J., 780:60, 2014b.

W. R. Johnston. Binary Minor Planets V7.0. NASA Planetary Data System, 219, 2014.

A. Kryszczyńska, F. Colas, P. Descamps, P. Bartczak, M. Polińska, T. Kwiatkowski, J. Lecacheux, R. Hirsch, M. Fagas, 
K. Kamiński, T. Michałowski, and A. Marciniak. New binary asteroid 809 Lundia. I. Photometry and modelling. Astron. Astrophys., 501:769-776, 2009.

C. Laver, I. de Pater, F. Marchis, M. Ádámkovics, and M. H. Wong. Component-resolved nearinfrared spectra of the (22) Kalliope system. Icarus, 204:574-579, December 2009.

H. F. Levison, W. F. Bottke, M. Gounelle, A. Morbidelli, D. Nesvorný, and K. Tsiganis. Contamination of the asteroid belt by primordial trans-Neptunian objects. Nature, 460:364366, 2009.

C. Magri, S. J. Ostro, D. J. Scheeres, M. C. Nolan, J. D. Giorgini, L. A. M. Benner, and J. L. Margot. Radar observations and a physical model of Asteroid 1580 Betulia. Icarus, 186:152177, 2007.

F. Marchis, P. Descamps, D. Hestroffer, and J. Berthier. Discovery of the triple asteroidal system 87 Sylvia. Nature, 463:822-824, 2005a.

F. Marchis, P. Descamps, D. Hestroffer, J. Berthier, M. E. Brown, and J. L. Margot. Satellites of (87) Sylvia. IAU Circ., 8582, 2005b.

F. Marchis, D. Hestroffer, P. Descamps, J. Berthier, A. H. Bouchez, R. D. Campbell, J. C. Y. Chin, M. A. van Dam, S. K. Hartman, E. M. Johansson, R. E. Lafon, D. Le Mignant, I. de Pater, P. J. Stomski, D. M. Summers, F. Vachier, P. L. Wizinovich, and M. H. Wong. A low density of $0.8 \mathrm{~g} \mathrm{~cm}^{-3}$ for the Trojan binary asteroid 617 Patroclus. Nature, 439:565-567, 2006a.

F. Marchis, M. H. Wong, J. Berthier, P. Descamps, D. Hestroffer, F. Vachier, D. Le Mignant, W. M. Keck Observatory, and I. de Pater. S/2006 (624) 1. IAU Circ., 8732, 2006b.
F. Marchis, M. Baek, P. Descamps, J. Berthier, D. Hestroffer, and F. Vachier. S/2004 (45) 1. IAU Circ., 8817, 2007.

F. Marchis, P. Descamps, M. Baek, A. W. Harris, M. Kaasalainen, J. Berthier, D. Hestroffer, and F. Vachier. Main belt binary asteroidal systems with circular mutual orbits. Icarus, 196:97118, 2008a.

F. Marchis, P. Descamps, J. Berthier, and J. P. Emery. S/2008 (216) 1 and S/2008 (216) 2. IAU Circ., 8980, 2008b.

F. Marchis, P. Descamps, J. Berthier, D. Hestroffer, F. Vachier, M. Baek, A. W. Harris, and D. Nesvorný. Main belt binary asteroidal systems with eccentric mutual orbits. Icarus, 195: 295-316, 2008c.

F. Marchis, J. Pollock, P. Pravec, M. Baek, J. Greene, L. Hutton, P. Descamps, D. E. Reichart, K. M. Ivarsen, J. A. Crain, M. C. Nysewander, A. P. Lacluyze, J. B. Haislip, and J. S. Harvey. (3749) Balam. IAU Circ., 8928, 2008d.

F. Marchis, B. Macomber, J. Berthier, F. Vachier, and J. P. Emery. S/2009 (93) 1 and S/2009 (93) 2. IAU Circ., 9069, 2009.

F. Marchis, V. Lainey, P. Descamps, J. Berthier, M. Van Dam, I. de Pater, B. Macomber, M. Baek, D. Le Mignant, H. B. Hammel, M. Showalter, and F. Vachier. A dynamical solution of the triple asteroid system (45) Eugenia. Icarus, 210:635-643, 2010.

F. Marchis, J. E. Enriquez, J. P. Emery, J. Berthier, P. Descamps, and F. Vachier. The origin of (90) Antiope from componentresolved near-infrared spectroscopy. Icarus, 213:252-264, 2011.

F. Marchis, J. E. Enriquez, J. P. Emery, M. Mueller, M. Baek, J. Pollock, M. Assafin, R. Vieira Martins, J. Berthier, F. Vachier, D. P. Cruikshank, L. F. Lim, D. E. Reichart, K. M. Ivarsen, J. B. Haislip, and A. P. LaCluyze. Multiple asteroid systems: Dimensions and 
thermal properties from Spitzer Space Telescope and ground-based observations. Icarus, 221:1130-1161, 2012.

F. Marchis, J. Durech, J. Castillo-Rogez, F. Vachier, M. Cuk, J. Berthier, M. H. Wong, P. Kalas, G. Duchene, M. A. van Dam, H. Hamanowa, and M. Viikinkoski. The Puzzling Mutual Orbit of the Binary Trojan Asteroid (624) Hektor. Astrophys. J. Lett., 783:L37, 2014.

J. L. Margot. S/2003 (379) 1. IAU Circ., 8182, 2003.

J. L. Margot. Recent observations of binary and multiple systems. In Second Workshop on Binaries in the Solar System, Wasowo, Poland, 2010.

J. L. Margot and M. E. Brown. Discovery and characterization of binary asteroids 22 Kalliope and 87 Sylvia. In Bull. Am. Astron. Soc., volume 33, 2001.

J. L. Margot and M. E. Brown. A low density M-type asteroid in the main belt. Science, 300 (5627):1939-1942, 2003.

J. L. Margot, M. C. Nolan, L. A. M. Benner, S. J. Ostro, R. F. Jurgens, J. D. Giorgini, M. A. Slade, and D. B. Campbell. Binary asteroids in the near-earth object population. Science, 296:1445-8, 2002.

J. L. Margot, P. Pravec, M. C. Nolan, E. S. Howell, L. A. M. Benner, J. D. Giorgini, R. F. Jurgens, S. J. Ostro, M. A. Slade, C. Magri, P. A. Taylor, P. D. Nicholson, and D. B. Campbell. Hermes as an exceptional case among binary near-earth asteroids. In IAU General Assembly, 2006.

F. Marzari, A. Rossi, and D. J. Scheeres. Combined effect of YORP and collisions on the rotation rate of small Main Belt asteroids. Icarus, 214(2):622-631, 2011.

J. McMahon and D. Scheeres. Secular orbit variation due to solar radiation effects: a detailed model for BYORP. Cel. Mech. Dyn. Astr., 106: 261-300, 2010a.

J. McMahon and D. Scheeres. Detailed prediction for the BYORP effect on binary near-Earth Asteroid (66391) $1999 \mathrm{KW} 4$ and implications for the binary population. Icarus, 209:494-509, $2010 b$.

W. J. Merline, L. M. Close, C. Dumas, C. R. Chapman, F. Roddier, F. Menard, D. C. Slater, G. Duvert, C. Shelton, and T. Morgan. Discovery of a moon orbiting the asteroid 45 Eugenia. Nature, 401:565+, 1999.

W. J. Merline, L. M. Close, J. C. Shelton, C. Dumas, F. Menard, C. R. Chapman, and D. C. Slater. Satellites of Minor Planets. IAU Circ., 7503, 2000.

W. J. Merline, L. M. Close, N. Siegler, D. Potter, C. R. Chapman, C. Dumas, F. Menard, D. C. Slater, A. C. Baker, M. G. Edmunds, G. Mathlin, O. Guyon, and K. Roth. S/2001 (617) 1. IAU Circ., 7741, 2001.

W. J. Merline, L. M. Close, N. Siegler, C. Dumas, C. Chapman, F. Rigaut, F. Menard, W. M. Owen, and D. C. Slater. S/2002 (3749) 1. IAU Circ., 7827, 2002a.

W. J. Merline, P. M. Tamblyn, C. Dumas, L. M. Close, C. R. Chapman, F. Menard, W. M. Owen, D. C. Slater, and J. Pepin. S/2002 (121) 1. IAU Circ., 7980, 2002 b.

W. J. Merline, S. J. Weidenschilling, D. D. Durda, J. L. Margot, P. Pravec, and A.D. Storrs. Asteroids Do Have Satellites. In W. F. Bottke, A. Cellino, P. Paolicchi, and R. P. Binzel, editors, Asteroids III, pages 289-312. Univ. of Arizona Press, 2002c.

W. J. Merline, L. M. Close, P. M. Tamblyn, F. Menard, C. R. Chapman, C. Dumas, G. Duvert, W. M. Owen, D. C. Slater, and M. F. Sterzik. S/2003 (1509) 1. IAU Circ., 8075, 2003a. 
W. J. Merline, P. M. Tamblyn, C. R. Chapman, D. Nesvorny, D. D. Durda, C. Dumas, A. D. Storrs, L. M. Close, and F. Menard. S/2003 (22899) 1. IAU Circ., 8232, 2003 b.

W. J. Merline, P. M. Tamblyn, C. Dumas, L. M. Close, C. R. Chapman, and F. Menard. S/2003 (130) 1. IAU Circ., 8183, 2003c.

W. J. Merline, P. M. Tamblyn, C. Dumas, F. Menard, L. M. Close, C. R. Chapman, G. Duvert, and N. Ageorges. S/2004 (4674) 1. IAU Circ., 8297, 2004.

W. J. Merline, P. M. Tamblyn, J. D. Drummond, J. C. Christou, A. R. Conrad, W. M. Keck Observatory, B. Carry, C. R. Chapman, C. Dumas, D. D. Durda, W. M. Owen, and B. L. Enke. S/2009 (317) 1. IAU Circ., 9099, 2009.

W. J. Merline, P. M. Tamblyn, B. D. Warner, P. Pravec, J. P. Tamblyn, C. Neyman, A. R. Conrad, W. M. Owen, B. Carry, J. D. Drummond, C. R. Chapman, B. L. Enke, W. M. Grundy, C. Veillet, S. B. Porter, C. Arcidiacono, J. C. Christou, D. D. Durda, A. W. Harris, H. A. Weaver, C. Dumas, D. Terrell, and P. Maley. S/2012 (2577) 1. IAU Circ., 9267, 2013.

T. Michałowski, P. Bartczak, F. P. Velichko, A. Kryszczyńska, T. Kwiatkowski, S. Breiter, F. Colas, S. Fauvaud, A. Marciniak, J. Michałowski, R. Hirsch, R. Behrend, L. Bernasconi, C. Rinner, and S. Charbonnel. Eclipsing binary asteroid 90 Antiope. Astron. Astrophys., 423:1159-1168, 2004.

A. Morbidelli, H. F. Levison, K. Tsiganis, and R. Gomes. Chaotic capture of Jupiter's Trojan asteroids in the early Solar System. Nature, 435:462-465, 2005.

N. A. Moskovitz. Colors of dynamically associated asteroid pairs. Icarus, 221(1):63-71, 2012.

M. Mueller, F. Marchis, J. P. Emery, A. W. Harris, S. Mottola, D. Hestroffer, J. Berthier, and
M. di Martino. Eclipsing binary Trojan asteroid Patroclus: Thermal inertia from Spitzer observations. Icarus, 205:505-515, 2010.

S. P. Naidu and J. L. Margot. Near-Earth Asteroid Satellite Spins Under Spin-Orbit Coupling. Astron. J., 149:80, 2015.

S. P. Naidu, J. L. Margot, M. W. Busch, P. A. Taylor, M. C. Nolan, E. S. Howell, J. D. Giorgini, L. A. M. Benner, M. Brozovic, and C. Magri. Dynamics of binary near-Earth asteroid system (35107) 1991 VH. In Bull. Am. Astron. Soc., volume 43, 2012.

S. P. Naidu, J. L. Margot, P. A. Taylor, M. C. Nolan, M. W. Busch, L. A. M. Benner, M. Brozovic, J. D. Giorgini, J. S. Jao, and C. Magri. Radar Imaging and Characterization of the Binary Near-Earth Asteroid (185851) 2000 DP107. Astron. J., 150:54, 2015.

D. Nesvorný, A. N. Youdin, and D. C. Richardson. Formation of Kuiper Belt Binaries by Gravitational Collapse. Astron. J., 140:785793, 2010.

M. C. Nolan, A. A. Hine, E. S. Howell, L. A. M. Benner, and J. D. Giorgini. $2003 \mathrm{SS}_{84}$. IAU Circ., 8220, 2003.

M. C. Nolan, E. S. Howell, T. M. Becker, C. Magri, J. D. Giorgini, and J. L. Margot. Arecibo Radar Observations of 2001 SN263: A NearEarth Triple Asteroid System. In Bull. Am. Astron. Soc., volume 40, 2008.

M. C. Nolan, C. Magri, E. S. Howell, L. A. M. Benner, J. D. Giorgini, C. W. Hergenrother, R. S. Hudson, D. S. Lauretta, J. L. Margot, S. J. Ostro, and D. J. Scheeres. Shape model and surface properties of the OSIRIS-REx target Asteroid (101955) Bennu from radar and lightcurve observations. Icarus, 226:629-640, 2013.

K. S. Noll, W. M. Grundy, E. I. Chiang, J. L. Margot, and S. D. Kern. Binaries in the Kuiper Belt. In A. Barucci, M. Boehnhardt, D. Cruikshank, and A. Morbidelli, editors, The Solar 
System Beyond Neptune, pages 345-363. Univ. of Arizona Press, 2008.

S. J. Ostro, J. L. Margot, L. A. M. Benner, J. D. Giorgini, D. J. Scheeres, E. G. Fahnestock, S. B. Broschart, J. Bellerose, M. C. Nolan, C. Magri, P. Pravec, P. Scheirich, R. Rose, R. F. Jurgens, E. M. De Jong, and S. Suzuki. Radar Imaging of Binary Near-Earth Asteroid (66391) 1999 KW4. Science, 314:1276-1280, 2006.

H. B. Perets and S. Naoz. Kozai Cycles, Tidal Friction, and the Dynamical Evolution of Binary Minor Planets. Astrophys. J. Lett., 699: L17-L21, 2009.

D. Polishook. Spin axes and shape models of asteroid pairs: Fingerprints of YORP and a path to the density of rubble piles. Icarus, 241:7996, 2014.

D. Polishook and N. Brosch. Photometry and spin rate distribution of small-sized main belt asteroids. Icarus, 199:319-332, 2009.

D. Polishook, N. Brosch, and D. Prialnik. Rotation periods of binary asteroids with large separations - Confronting the Escaping Ejecta Binaries model with observations. Icarus, 212: 167-174, 2011.

D. Polishook, N. Moskovitz, R. P. Binzel, F. E. DeMeo, D. Vokrouhlický, J. Žižka, and D. Oszkiewicz. Observations of "fresh" and weathered surfaces on asteroid pairs and their implications on the rotational-fission mechanism. Icarus, 233:9-26, 2014a.

D. Polishook, N. Moskovitz, F. E. DeMeo, and R. P. Binzel. Rotationally resolved spectroscopy of asteroid pairs: No spectral variation suggests fission is followed by settling of dust. Icarus, 243:222-235, $2014 \mathrm{~b}$.

P. Pravec and G. Hahn. Two-Period Lightcurve of $1994 \mathrm{AW}_{1}$ : Indication of a Binary Asteroid? Icarus, 127:431-440, 1997.
P. Pravec and A. W. Harris. Fast and Slow Rotation of Asteroids. Icarus, 148:12-20, 2000.

P. Pravec and A. W. Harris. Binary asteroid population. 1. Angular momentum content. Icarus, 190:250-259, 2007.

P. Pravec and D. Vokrouhlický. Significance analysis of asteroid pairs. Icarus, 204:580-588, 2009.

P. Pravec, M. Wolf, and L. Šarounová. How many binaries are there among the near-Earth asteroids? In J. Svoren, E. M. Pittich, and H. Rickman, editors, IAU Colloq. 173: Evolution and Source Regions of Asteroids and Comets, page 159, 1999.

P. Pravec, P. Scheirich, P. Kušnirák, L. Šarounová, S. Mottola, G. Hahn, P. Brown, G. Esquerdo, N. Kaiser, Z. Krzeminski, D. P. Pray, B. D. Warner, A. W. Harris, M. C. Nolan, E. S. Howell, L. A. M. Benner, J. L. Margot, A. Galád, W. Holliday, M. D. Hicks, Y. N. Krugly, D. Tholen, R. Whiteley, F. Marchis, D. R. Degraff, A. Grauer, S. Larson, F. P. Velichko, W. R. Cooney, R. Stephens, J. Zhu, K. Kirsch, R. Dyvig, L. Snyder, V. Reddy, S. Moore, Š. Gajdoš, J. Világi, G. Masi, D. Higgins, G. Funkhouser, B. Knight, S. Slivan, R. Behrend, M. Grenon, G. Burki, R. Roy, C. Demeautis, D. Matter, N. Waelchli, Y. Revaz, A. Klotz, M. Rieugné, P. Thierry, V. Cotrez, L. Brunetto, and G. Kober. Photometric survey of binary near-Earth asteroids. Icarus, 181:63-93, 2006.

P. Pravec, A. W. Harris, and B. D. Warner. NEA rotations and binaries. In IAU Symposium, volume 236, pages 167-176, 2007.

P. Pravec, A. W. Harris, D. Vokrouhlický, B. D. Warner, P. Kušnirák, K. Hornoch, D. P. Pray, D. Higgins, J. Oey, A. Galád, Š. Gajdoš, L. Kornoš, J. Világi, M. Husárik, Y. N. Krugly, V. Shevchenko, V. Chiorny, N. Gaftonyuk, W. R. Cooney, J. Gross, D. Terrell, R. D. Stephens, R. Dyvig, V. Reddy, J. G. Ries, F. Colas, J. Lecacheux, R. Durkee, G. Masi, 
R. A. Koff, and R. Goncalves. Spin rate distribution of small asteroids. Icarus, 197:497504, 2008.

P. Pravec, D. Vokrouhlický, D. Polishook, D. J. Scheeres, A. W. Harris, A. Galád, O. Vaduvescu, F. Pozo, A. Barr, P. Longa, F. Vachier, F. Colas, D. P. Pray, J. Pollock, D. Reichart, K. Ivarsen, J. Haislip, A. Lacluyze, P. Kušnirák, T. Henych, F. Marchis, B. Macomber, S. A. Jacobson, Y. N. Krugly, A. V. Sergeev, and A. Leroy. Formation of asteroid pairs by rotational fission. Nature, 466:10851088, 2010.

P. Pravec, P. Scheirich, D. Vokrouhlický, A. W. Harris, P. Kušnirák, K. Hornoch, D. P. Pray, D. Higgins, A. Galád, J. Világi, Š. Gajdoš, L. Kornoš, J. Oey, M. Husárik, W. R. Cooney, J. Gross, D. Terrell, R. Durkee, J. Pollock, D. E. Reichart, K. Ivarsen, J. Haislip, A. LaCluyze, Y. N. Krugly, N. Gaftonyuk, R. D. Stephens, R. Dyvig, V. Reddy, V. Chiorny, O. Vaduvescu, P. Longa-Peña, A. Tudorica, B. D. Warner, G. Masi, J. Brinsfield, R. Gonçalves, P. Brown, Z. Krzeminski, O. Gerashchenko, V. Shevchenko, I. Molotov, and F. Marchis. Binary asteroid population. 2. Anisotropic distribution of orbit poles of small, inner main-belt binaries. Icarus, 218: 125-143, 2012.

P. Pravec, P. Kusnirak, K. Hornoch, A. Galad, Y. N. Krugly, V. Chiorny, R. Inasaridze, O. Kvaratskhelia, V. Ayvazian, O. Parmonov, J. Pollock, S. Mottola, J. Oey, D. Pray, J. Zizka, J. Vrastil, I. Molotov, D. E. Reichart, K. M. Ivarsen, J. B. Haislip, and A. LaCluyze. (8306) Shoko. IAU Circ., 9268, 2013.

P. Rojo and J. L. Margot. S/2007 (702) 1. CBET, 1016, 2007.

A. Rossi, F. Marzari, and D. J. Scheeres. Computing the effects of YORP on the spin rate distribution of the NEO population. Icarus, 202(1): 95-103, 2009.
B. Rozitis, E. Maclennan, and J. P. Emery. Cohesive forces prevent the rotational breakup of rubble-pile asteroid (29075) 1950 DA. Nature, 512:174-176, 2014.

D. P. Rubincam. Radiative Spin-up and Spindown of Small Asteroids. Icarus, 148(1):2-11, 2000 .

D. P. Sánchez and D. J. Scheeres. Simulating Asteroid Rubble Piles with A Self-gravitating Soft-sphere Distinct Element Method Model. Astrophys. J., 727(2):120, 2011.

D. P. Sánchez and D. J. Scheeres. The strength of regolith and rubble pile asteroids. Meteoritics \& Planetary Science, 49(5):788-811, 2014.

D. J. Scheeres. Stability of Binary Asteroids. Icarus, 159:271-283, October 2002.

D. J. Scheeres. Rotational fission of contact binary asteroids. Icarus, 189:370-385, 2007.

D. J. Scheeres. Stability of the planar full 2-body problem. Cel. Mech. Dyn. Astr., 104:103-128, 2009a.

D. J. Scheeres. Minimum energy asteroid reconfigurations and catastrophic disruptions. Planetary and Space Science, 57(2):154-164, 2009b.

D. J. Scheeres, E. G. Fahnestock, S. J. Ostro, J. L. Margot, L. A. M. Benner, S. B. Broschart, J. Bellerose, J. D. Giorgini, M. C. Nolan, C. Magri, P. Pravec, P. Scheirich, R. Rose, R. F. Jurgens, E. M. De Jong, and S. Suzuki. Dynamical Configuration of Binary Near-Earth Asteroid (66391) 1999 KW4. Science, 314:1280-1283, 2006.

P. Scheirich and P. Pravec. Modeling of lightcurves of binary asteroids. Icarus, 200: 531-547, 2009.

P. Scheirich, P. Pravec, S. A. Jacobson, J. Ďurech, P. Kušnirák, K. Hornoch, S. Mottola, M. Mommert, S. Hellmich, D. Pray, D. Polishook, Y. N. Krugly, R. Y. Inasaridze, 
O. I. Kvaratskhelia, V. Ayvazian, I. Slyusarev, J. Pittichová, E. Jehin, J. Manfroid, M. Gillon, A. Galád, J. Pollock, J. Licandro, V. AlíLagoa, J. Brinsfield, and I. E. Molotov. The binary near-Earth Asteroid (175706) $1996 \mathrm{FG}_{3}$ An observational constraint on its orbital evolution. Icarus, 245:56-63, 2015.

M. K. Shepard, J. L. Margot, C. Magri, M. C. Nolan, J. Schlieder, B. Estes, S. J. Bus, E. L. Volquardsen, A. S. Rivkin, L. A. M. Benner, J. D. Giorgini, S. J. Ostro, and M. W. Busch. Radar and infrared observations of binary near-Earth Asteroid 2002 CE26. Icarus, 184:198-210, 2006.

A. Springmann, P. A. Taylor, E. S. Howell, M. C. Nolan, L. A. M. Benner, M. Brozović, J. D. Giorgini, and J. L. Margot. Radar shape model of binary near-Earth asteroid (285263) 1998 $\mathrm{QE}_{2}$. In Lunar and Planetary Science Conference, volume 45, 2014.

Y. Takahashi, M. W. Busch, and D. J. Scheeres. Spin State and Moment of Inertia Characterization of 4179 Toutatis. Astron. J., 146:95, 2013.

P. M. Tamblyn, W. J. Merline, C. R. Chapman, D. Nesvorny, D. D. Durda, C. Dumas, A. D. Storrs, L. M. Close, and F. Menard. S/2004 (17246) 1. IAU Circ., 8293, 2004.

P. Tanga and M. Delbo. Asteroid occultations today and tomorrow: toward the GAIA era. Astron. Astrophys., 474:1015-1022, 2007.

P. A. Taylor and J. L. Margot. Tidal evolution of close binary asteroid systems. Cel. Mech. Dyn. Astr., 108:315-338, 2010.

P. A. Taylor and J. L. Margot. Binary asteroid systems: Tidal end states and estimates of material properties. Icarus, 212:661-676, 2011.

P. A. Taylor and J. L. Margot. Tidal end states of binary asteroid systems with a nonspherical component. Icarus, 229:418-422, 2014.
P. A. Taylor, J. L. Margot, M. C. Nolan, L. A. M. Benner, S. J. Ostro, J. D. Giorgini, and C. Magri. The shape, mutual orbit, and tidal evolution of binary near-Earth asteroid 2004 DC. In Asteroids, Comets, Meteors Conference, volume 1405, page 8322, 2008.

P. A. Taylor, E. S. Howell, M. C. Nolan, and A. A. Thane. The shape and spin distributions of near-Earth asteroids observed with the Arecibo radar system. In Bull. Am. Astron. Soc., volume 44, 2012a.

P. A. Taylor, M. C. Nolan, and E. S. Howell. 5143 Heracles. CBET, 3176, 2012 b.

P. A. Taylor, M. C. Nolan, E. S. Howell, L. A. M. Benner, M. Brozovic, J. D. Giorgini, J. L. Margot, M. W. Busch, S. P. Naidu, C. Nugent, C. Magri, and M. K. Shepard. $2004 \mathrm{FG}_{11}$. CBET, 3091, 2012c.

P. A. Taylor, E. S. Howell, M. C. Nolan, A. Springmann, M. Brozovic, L. M. Benner, J. S. Jao, J. D. Giorgini, J. Margot, J. Fang, T. M. Becker, Y. R. Fernandez, R. J. Vervack, P. Pravec, P. Kusnirak, L. Franco, A. Ferrero, A. Galad, D. P. Pray, B. D. Warner, and M. D. Hicks. Physical characterization of binary near-Earth asteroid (153958) $2002 \mathrm{AM}_{31}$. In Bull. Am. Astron. Soc., volume 45, 2013.

P. A. Taylor, B. D. Warner, C. Magri, A. Springmann, M. C. Nolan, E. S. Howell, K. J. Miller, L. F. Zambrano-Marin, J. E. Richardson, M. Hannan, and P. Pravec. The smallest binary asteroid? The discovery of equal-mass binary 1994 CJ $_{1}$. In Bull. Am. Astron. Soc., volume 46, 2014.

E. F. Tedesco. Binary asteroids - Evidence for their existence from lightcurves. Science, 203: 905-907, 1979.

B. Timerson, J. Brooks, S. Conard, D. W. Dunham, D. Herald, A. Tolea, and F. Marchis. Occultation evidence for a satellite of the Trojan asteroid (911) Agamemnon. Planetary and Space Science, 87:78-84, 2013. 
D. Vokrouhlický. (3749) Balam: A Very Young Multiple Asteroid System. Astrophys. J. Lett., 706:L37-L40, 2009.

D. Vokrouhlický and D. Nesvorný. Pairs of Asteroids Probably of a Common Origin. Astron. J., 136:280-290, 2008.

K. J. Walsh, D. C. Richardson, and P. Michel. Rotational breakup as the origin of small binary asteroids. Nature, 454:188-191, 2008.

S. J. Weidenschilling. Hektor - Nature and origin of a binary asteroid. Icarus, 44:807-809, 1980.

S. J. Weidenschilling, P. Paolicchi, and V. Zappalà. Do asteroids have satellites? In R. P. Binzel, T. Gehrels, and M. S. Matthews, editors, Asteroids II, pages 643-660. University of Arizona Press, 1989.

S. J. Weidenschilling, F. Marzari, D. R. Davis, and C. Neese. Origin of the Double Asteroid 90 Antiope: A Continuing Puzzle. In Lunar and Planetary Science Conference, volume 32, page 1890, 2001.

S. D. Wolters, P. R. Weissman, A. Christou, S. R. Duddy, and S. C. Lowry. Spectral similarity of unbound asteroid pairs. Mon. Not. R. Astron. Soc., 439:3085-3093, 2014.

D. K. Yeomans, P. G. Antreasian, A. Cheng, D. W. Dunham, R. W. Farquhar, R. W. Gaskell, J. D. Giorgini, C. E. Helfrich, A. S. Konopliv, J. V. McAdams, J. K. Miller, W. M. Owen, Jr., P. C. Thomas, J. Veverka, and B. G. Williams. Estimating the mass of asteroid 433 Eros during NEAR spacecraft flyby. Science, 285:560$561,1999$. 